\title{
Tracing early evolutionary stages of high-mass star formation with molecular lines
}

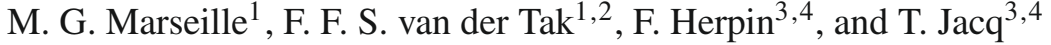 \\ 1 SRON Netherlands Institute for Space Research, Landleven 12, 9747 AD Groningen, The Netherlands \\ e-mail: [marseille;vdtak]@sron.nl \\ 2 Kapteyn Astronomical Institute, PO Box 800, 9700 AV Groningen, The Netherlands \\ 3 Université Bordeaux 1, Laboratoire d'Astrophysique de Bordeaux, 2 rue de l'Observatoire, BP 89, 33271 Floirac Cedex, France \\ e-mail: [herpin; jacq]@obs.u-bordeaux1.fr \\ ${ }^{4}$ CNRS/INSU, UMR 5804, BP 89, 33271 Floirac Cedex, France
}

Received 27 October 2009 / Accepted 11 June 2000

ABSTRACT

\begin{abstract}
Context. Despite its major role in the evolution of the interstellar medium, the formation of high-mass stars $\left(M \geq 10 M_{\odot}\right)$ remains poorly understood. Two types of massive star cluster precursors, the so-called massive dense cores (MDCs), have been observed, which differ in terms of their mid-infrared brightness. The origin of this difference has not yet been established and may be the result of evolution, density, geometry differences, or a combination of these.

Aims. We compare several molecular tracers of physical conditions (hot cores, shocks) observed in a sample of mid-IR weakly emitting MDCs with previous results obtained in a sample of exclusively mid-IR bright MDCs. We attempt to understand the differences between these two types of object.

Methods. We present single-dish observations of $\mathrm{HDO}, \mathrm{H}_{2}^{18} \mathrm{O}, \mathrm{SO}_{2}$, and $\mathrm{CH}_{3} \mathrm{OH}$ lines at $\lambda=1.3-3.5 \mathrm{~mm}$. We study line profiles and estimate abundances of these molecules, and use a partial correlation method to search for trends in the results.

Results. The detection rates of thermal emission lines are found to be very similar for both mid-IR quiet and bright objects. The abundances of $\mathrm{H}_{2} \mathrm{O}$, HDO ( $10^{-13}$ to $10^{-9}$ in the cold outer envelopes), $\mathrm{SO}_{2}$ and $\mathrm{CH}_{3} \mathrm{OH}$ differ from source to source but independently of their mid-IR flux. In contrast, the methanol class I maser emission, a tracer of outflow shocks, is found to be strongly anti-correlated with the $12 \mu \mathrm{m}$ source brightnesses.

Conclusions. The enhancement of the methanol maser emission in mid-IR quiet MDCs may be indicative of a more embedded nature. Since total masses are similar between the two samples, we suggest that the matter distribution is spherical around mid-IR quiet sources but flattened around mid-IR bright ones. In contrast, water emission is associated with objects containing a hot molecular core, irrespective of their mid-IR brightness. These results indicate that the mid-IR brightness of MDCs is an indicator of their evolutionary stage.
\end{abstract}

Key words. ISM: abundances - evolution - stars: formation - line: profiles

\section{Introduction}

High-mass stars play a significant role in shaping their host galaxies, because of their high UV luminosity and mechanical input (wind shocks and supernovæ), but their formation remains poorly understood. The scaling-up of existing models of lowmass star formation (Shu et al. 1993) is unhelpful, because radiation pressure would halt the accretion (e.g. Yorke \& Sonnhalter 2002) leading to a final stellar mass of $10 M_{\odot}$ at most. To allow the formation of more massive stars, the quasi-static scenario has been modified by increasing the mass accretion rate and including turbulence or energetic dynamics (McKee \& Tan 2003; Henriksen et al. 1997). Other approaches, such as competitive accretion in massive protostar clusters (Bonnell et al. 1997, 2001) or protostellar mergers (Bonnell et al. 1998) may also provide an answer to this problem. The physical conditions in highmass star formation sites indeed correspond to a dense and turbulent medium where a monolithic infall onto a single massive protostar is not expected. As an example, observed molecular emission line widths are always dominated by turbulent velocities $\left(v_{\mathrm{T}}>0.5 \mathrm{~km} \mathrm{~s}^{-1}\right.$, e.g., Sridharan et al. 2002), and are observed from large to small scales (see $\mathrm{C}^{34} \mathrm{~S}$ lines observed by
Leurini et al. 2007a, with interferometry in IRAS 05358+3543), and are greater than the speed of sound $\left(a_{\mathrm{s}} \simeq 0.3 \mathrm{~km} \mathrm{~s}^{-1}\right)$. In addition, Jeans masses of massive star formation regions are very low compared to their own masses $\left(2.5 M_{\odot}\right.$ for a mean temperature of $20 \mathrm{~K}$, compared to few hundred solar masses, e.g. the Cygnus-X sample by Motte et al. 2007), implying that a high level of fragmentation may play an important role. On the other hand, the magnetic field could limit this fragmentation (WardThompson et al. 2007; Girart et al. 2009). A scenario of clustered massive star formation is now favoured, but its major steps have yet to be defined with new observations and studies.

Searches for early stages of massive star formation have revealed a class of objects, called high-mass protostellar objects (hereafter HMPOs) in the sample of Molinari et al. (1996) and Sridharan et al. (2002), which are relatively extended $(\sim 1 \mathrm{pc})$, and contain one or several clumps called massive dense cores (hereafter MDCs), as seen in the Cygnus-X sample by Motte et al. (2007). The main characteristics of MDCs are weak radio emission from ionized gas (less than a few $10 \mathrm{mJy}$ at $2.5 \mathrm{~cm}$ ) despite a high total luminosity $\left(L>10^{3} L_{\odot}\right.$ ), a high mass ( $>$ few hundreds of solar masses), and a high density $\left(n>10^{5} \mathrm{~cm}^{-3}\right)$ in a typical size of $0.1 \mathrm{pc}(\sim 20000 \mathrm{AU})$. They exhibit evidence 
Table 1. Source sample.

\begin{tabular}{|c|c|c|c|c|c|c|c|c|c|c|c|}
\hline 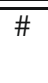 & Source & $\begin{array}{c}\alpha \\
(\mathrm{J} 2000)\end{array}$ & $\begin{array}{c}\delta \\
(\mathrm{J} 2000)\end{array}$ & $\begin{array}{c}v_{\text {lsr }} \\
\left(\mathrm{km} \mathrm{s}^{-1}\right)\end{array}$ & $\begin{array}{c}D \\
(\mathrm{kpc})\end{array}$ & $\begin{array}{c}L \\
\left(\times 10^{4} L_{\odot}\right)\end{array}$ & $\begin{array}{c}M \\
\left(M_{\odot}\right)\end{array}$ & $\begin{array}{c}r \\
(\mathrm{pc})\end{array}$ & $\begin{array}{c}\mathrm{CH}_{3} \mathrm{OH} / \mathrm{H}_{2} \mathrm{O} \\
\text { masers }\end{array}$ & $\begin{array}{c}L_{12 \mu \mathrm{m}} \\
(\mathrm{Jy})\end{array}$ & Ref. \\
\hline 1 & IRAS $05358+3543$ & $05^{\mathrm{h}} 39^{\mathrm{m}} 10.4^{\mathrm{s}}$ & $+35^{\circ} 45^{\prime} 19^{\prime \prime}$ & -17.6 & 1.8 & 0.7 & 400 & 0.10 & yes/yes & 6.29 & 1,2 \\
\hline 2 & IRAS 18089-1732 & $18^{\mathrm{h}} 11^{\mathrm{m}} 51.5^{\mathrm{s}}$ & $-17^{\circ} 31^{\prime} 29^{\prime \prime}$ & +33.8 & 3.6 & 3.2 & 1000 & 0.14 & yes/yes & 27.27 & 1,3 \\
\hline 3 & IRAS 18151-1208 MM1 & $18^{\mathrm{h}} 17^{\mathrm{m}} 58.0^{\mathrm{s}}$ & $-12^{\circ} 07^{\prime} 27^{\prime \prime}$ & +33.4 & 3.0 & 1.4 & 330 & 0.13 & yes/no & 67.89 & 4 \\
\hline 4 & IRAS 18151-1208 MM2 & $18^{\mathrm{h}} 17^{\mathrm{m}} 50.4^{\mathrm{s}}$ & $-12^{\circ} 07^{\prime} 55^{\prime \prime}$ & +29.7 & 3.0 & 0.3 & 230 & 0.11 & no/yes & 0.65 & 4 \\
\hline 5 & IRAS 18264-1152 & $18^{\mathrm{h}} 29^{\mathrm{m}} 14.3^{\mathrm{s}}$ & $-11^{\circ} 50^{\prime} 23^{\prime \prime}$ & +43.6 & 3.5 & 1.4 & 1200 & 0.13 & yes/yes & 12.25 & $1,2,5$ \\
\hline 6 & W43-MM1 & $18^{\mathrm{h}} 47^{\mathrm{m}} 47.0^{\mathrm{s}}$ & $-01^{\circ} 54^{\prime} 28^{\prime \prime}$ & +98.8 & 5.5 & 2.3 & 2000 & 0.12 & yes/yes & 11.51 & 2,6 \\
\hline 7 & $\mathrm{DR} 21(\mathrm{OH})$ & $20^{\mathrm{h}} 39^{\mathrm{m}} 00.8^{\mathrm{s}}$ & $+42^{\circ} 22^{\prime} 48^{\prime \prime}$ & -4.5 & 1.7 & 0.2 & 450 & 0.13 & $\cdots$ & 3.10 & 7 \\
\hline 8 & DR21(OH)-S & $20^{\mathrm{h}} 39^{\mathrm{m}} 01.5^{\mathrm{s}}$ & $+42^{\circ} 22^{\prime} 04^{\prime \prime}$ & -3.1 & 1.7 & 0.4 & 200 & 0.13 & $\ldots$ & 0.88 & 7 \\
\hline 9 & NGC 7538S & $23^{\mathrm{h}} 13^{\mathrm{m}} 44.5^{\mathrm{s}}$ & $+61^{\circ} 26^{\prime} 50^{\prime \prime}$ & -57.0 & 2.8 & 1.3 & 400 & 0.14 & yes/yes & 4.61 & 8,9 \\
\hline 10 & IRAS $23385+6053$ & $23^{\mathrm{h}} 40^{\mathrm{m}} 53.3^{\mathrm{s}}$ & $+61^{\circ} 10^{\prime} 19^{\prime \prime}$ & -49.0 & 4.9 & 1.6 & 370 & 0.05 & .../yes & 41.96 & $1,6,10$ \\
\hline
\end{tabular}

Notes. Columns 2-10 present coordinates, velocities in the local standard of rest, heliocentric distances, luminosities, mass estimates and source sizes at millimetre wavelength of the sources, extracted from references given in the last column.

References. (1) Beuther et al. (2002a); (2) Herpin et al. (2009); (3) Leurini et al. (2007b); (4) Marseille et al. (2008); (5) Szymczak et al. (2000); Edris et al. (2007); (6) Molinari et al. (1996); (7) Motte et al. (2007); (8) Sandell et al. (2003); (9) Barvainis \& Deguchi (1989), Cernicharo et al. (1990), Haschick et al. (1989); (10) Molinari et al. (1998).

of accretion activity: molecular bipolar outflows (Beuther et al. 2002c), water and methanol masers (Beuther et al. 2002d), and large-scale inflows (Motte et al. 2003; Herpin et al. 2009). As reviewed by Zinnecker \& Yorke (2007), MDCs can be seen as proto-clusters that host the formation of high-mass stars.

MDCs are usually divided into mid-infrared "quiet" and "bright" sources (hereafter $\mathrm{mIRq}$ and mIRb sources). van der Tak et al. (2000a) define the division between them to be $10 \mathrm{Jy}$ (12 $\mu \mathrm{m}$ flux density), whereas Motte et al. (2007) assume a limit of $10 \mathrm{Jy}$ for the MSX flux densities at $21.6 \mu \mathrm{m}$, in accordance with the distance of Cygnus-X. These values are equivalent to the classical limit between class 0 and class I low-mass protostars obtained from the ratio of mid-IR to total luminosity (André et al. 1993), as MDCs have similar total luminosities. The link between $\mathrm{mIRq}$ and $\mathrm{mIRb}$ sources is not clear and the various mid-IR flux densities may be the consequence of different source orientations relative to the line of sight, or differences in the gas density distribution (van der Tak et al. 2006; Marseille et al. 2008). This last hypothesis is supported by the observation of developed hot molecular cores (HMCs hereafter) in MDCs (e.g. van der Tak et al. 2000b; Motte et al. 2003; Leurini et al. 2007b). On the other hand, chemical data indicate that $\mathrm{mIRq}$ sources have a lower temperature than bright ones (Marseille et al. 2008), which imply they represent a less evolved stage.

To more clearly define the evolutionary paths of $\mathrm{mIRq}$ and $\mathrm{mIRb}$ sources, this paper measures chemical, physical, and dynamical tracers in a sample of ten mid-IRq MDCs, extending the study by van der Tak et al. (2006) of mIRb-MDCs. We include water species ( $\mathrm{HDO}$ and $\mathrm{H}_{2}^{18} \mathrm{O}$ ), which can exhibit an abundance enhancement because of their release in the gas phase from the dust grain surfaces, by means of a hot core, or shocks induced by bipolar outflows (e.g. Cernicharo et al. 1990; van Dishoeck \& Helmich 1996; Harwit et al. 1998; Boonman et al. 2003). Thus we probe the processes critically related to the formation of massive stars. To further investigate this approach, we focus on to hot cores and shock tracers using observations of a high-energy transition of $\mathrm{SO}_{2}$ and a methanol class I maser. We apply statistical tools to our sample (correlation and partial correlation factors) to constrain biases and real trends in our results.

The paper is arranged as follows. Section 2 presents the source sample, and Sect. 3 the new observations of $\mathrm{HDO}, \mathrm{H}_{2}^{18} \mathrm{O}$, $\mathrm{SO}_{2}$ and $\mathrm{CH}_{3} \mathrm{OH}$ molecular transitions. Section 4 checks for detections and describes the line profiles. Section 5 treats the case of the methanol class I maser emission, extracting the maser emission from the thermal emission. In Sect. 6, a modelling method is applied to extract molecular abundances, themselves analysed in Sect. 7 using statistical methods to find biases and real correlations between them and the physical characteristics of MDCs. In Sect. 8, we discuss our results and present conclusions about the class I maser emission behaviour, describe the differences and similarities between mIRq- and mIRb-MDCs, and present new ideas about their evolutionary stages.

\section{Source sample}

We observed ten MDCs with a low mid-IR brightness (see Table 1; fluxes have been corrected to assume a single distance of $1.7 \mathrm{kpc}$ ), taken from well-known samples (Beuther et al. 2002b; Molinari et al. 1996; Motte et al. 2007). Their physical properties incorporate a large range of masses (200-2000 $M_{\odot}$ ) and luminosities (between $2 \times 10^{3}$ and $3 \times 10^{4} L_{\odot}$ ) within a typical size of $\sim 0.1$ pc. All are dense $\left(n_{\mathrm{H}_{2}} \simeq 10^{5}-10^{7} \mathrm{~cm}^{-3}\right)$, most of them $(8 \mathrm{in}$ total) exhibiting water and/or methanol class II masers, and bipolar outflows. Chosen MDCs are weak at $\mathrm{cm}$ wavelengths (except for IRAS 05358+3543, which exhibits an UC-HII region), making them good candidates to represent an early stage of the formation of massive stars. Other studies infer a high accretion rate for these sources $\left(\sim 10^{-3}-10^{-4} M_{\odot} \mathrm{yr}^{-1}\right)$ derived from the power of their molecular outflows, and a clustered formation process observed by interferometry (Zapata et al. 2006; Leurini et al. 2007a; Molinari et al. 2008; Bontemps et al. 2010). According to IRAS or MSX data, these sources emit between 0.2 and $22 \mathrm{Jy}$ at $12 \mu \mathrm{m}$. This sample is complementary to the one observed by van der Tak et al. (2006), which contained mIRb-MDCs exclusively.

\section{Observations}

We performed observations at the Pico Veleta with the IRAM $30 \mathrm{~m}$ antenna $^{1}$, in June 2006 and February 2009.

\footnotetext{
1 IRAM is an international institute for research in millimetre astronomy, co-founded by the Centre National de la Recherche Scientifique (France), the Max Planck Gesellschaft (Germany) and the Instituto Geografico Nacional (Spain).
} 
Early evolutionary stages of high-mass star formation: M. G. Marseille et al.

Table 2. Molecular lines observed.

\begin{tabular}{lcccccccc}
\hline \hline Species & $\begin{array}{c}\text { Transition } \\
J_{K_{p}, K_{o}}\end{array}$ & $\begin{array}{c}\text { Frequency } \\
(\mathrm{GHz})\end{array}$ & $\begin{array}{c}E_{\mathrm{up}} \\
(\mathrm{K})\end{array}$ & $\begin{array}{c}\text { HPBW } \\
\left({ }^{\prime \prime}\right)\end{array}$ & $\eta_{\mathrm{mb}}$ & Receiver & $\begin{array}{c}\delta v^{a} \\
\left(\mathrm{~m} \cdot \mathrm{s}^{-1}\right)\end{array}$ & $\begin{array}{c}T_{\text {sys }} \\
(\mathrm{K})\end{array}$ \\
\hline $\mathrm{HDO}$ & $1_{1,0} \rightarrow 1_{0,1}$ & 80.5783 & 47 & 31 & 0.79 & $\mathrm{~A} 100$ & 74 & $140-190$ \\
& $3_{1,2} \rightarrow 2_{2,1}$ & 225.8967 & 168 & 11 & 0.54 & $\mathrm{~B} 230$ & 53 & $750-1600$ \\
$\mathrm{H}_{2}^{18} \mathrm{O}$ & $3_{1,3} \rightarrow 2_{2,0}$ & 203.4075 & 204 & 12 & 0.57 & $\mathrm{~A} 230$ & 59 & $600-1300$ \\
$\mathrm{SO}_{2}$ & $12_{0,12} \rightarrow 11_{1,11}$ & 203.3916 & 70 & 12 & 0.57 & $\mathrm{~A} 230$ & 59 & $600-1300$ \\
$\mathrm{CH}_{3} \mathrm{OH}$ & $5_{-1,5} \rightarrow 4_{0,4} \mathrm{E}$ & 84.5212 & 40 & 29 & 0.78 & $\mathrm{~B} 100$ & 71 & $140-190$ \\
\hline
\end{tabular}

Notes. Columns 2-9 indicate energy level transitions, line emission rest frequencies, half power beam width (HPBW), main beam efficiency $\eta_{\mathrm{mb}}$, receiver name, velocity resolution $\delta v$, and system temperature $T_{\text {sys. }}{ }^{(a)}$ Values for the VESPA backend.

Observations were performed while the weather was good $\left(\tau_{0}^{\text {atm }} \sim 0.07-0.6\right.$ at $\left.225 \mathrm{GHz}\right)$. We achieved system temperatures of 150-190 K for lower frequency transitions and 600-1600 K for higher ones (see Table 2 for further details).

We used simultaneously the A100, A230, B100, and B230 receivers to cover five molecular emission lines: $\operatorname{HDO}\left(1_{1,0}-\right.$ $\left.1_{0,1}\right)$ and $\left(3_{1,2}-2_{2,1}\right)$ at $80.6 \mathrm{GHz}$ and $225.9 \mathrm{GHz}$ respectively, $\mathrm{CH}_{3} \mathrm{OH}\left(5_{1}-4_{0}\right)$ at $84.5 \mathrm{GHz}, \mathrm{H}_{2}^{18} \mathrm{O}\left(3_{1,3}-2_{2,0}\right)$, and $\mathrm{SO}_{2}\left(12_{0,12}-\right.$ $\left.11_{1,11}\right)$ at $203.4 \mathrm{GHz}$. We tuned the VESPA correlator to obtain spectral resolutions of 20 and $40 \mathrm{kHz}$, hence velocity resolutions of between 50 and $80 \mathrm{~m} \mathrm{~s}^{-1}$ (see Table 2 for details). We observed sources in a single point mode, pointing on the continuum peak emission at millimetre wavelengths. We chose the wobbler-switching observation mode as the sources are reasonably isolated, using a throw of $4^{\prime}$ to the west. Pointing and focus calibrations were set on Jupiter and Neptune. The pointing accuracy was of the order of $2^{\prime \prime}$.

We performed the data reduction with the software CLASS from the GILDAS suite (Guilloteau \& Lucas 2000). After subtracting linear or polynomial baselines, we summed spectra according to the position and frequency. We converted antenna temperature $T_{\mathrm{a}}^{*}$ into main beam temperature $T_{\mathrm{mb}}$ using the efficiency parameter $\eta_{\text {mb }}$ provided by IRAM (see Table 2 ). We measured the line parameters (central velocity, width, peak intensity, and line flux) by fitting one or multiple Gaussian profiles.

\section{Results}

\subsection{Spectral line detections}

The methanol emission is detected towards all sources at a signal-to-noise ratio of 15 or more, with a peak $T_{\mathrm{mb}}$ varying from 0.6 to $\sim 40 \mathrm{~K}$ (see Fig. 1). For all sources, we detect wings or multiple velocity components. Unlike $\mathrm{CH}_{3} \mathrm{OH}$, the $\mathrm{SO}_{2}$ lines are detected in 7 sources only and have a Gaussian shape. The $\mathrm{H}_{2}^{18} \mathrm{O}$ line is seen toward 3 sources, W43-MM1, DR21(OH), and IRAS 18089-1732; the line profiles exhibit a Gaussian shape, except for DR21(OH) where the observed signal is more complex (see Fig. 3 and following section). The HDO line emission at $1 \mathrm{~mm}$ and $3 \mathrm{~mm}$ is detected toward 4 sources: W43MM1, DR21(OH), IRAS 18089-1732, and NGC 7538S. The HDO lines are always either detected or both non-detected (see Fig. 2). We do not detect special features in the line profiles, except for DR21(OH) whose line profile is most closely fitted by a two-component velocity model. Measured parameters of single peaked lines are listed in Table 3. Methanol and other special features (such as the two velocity components in DR21(OH)) are treated in Sect. 4.2.

The $1.3 \mathrm{~mm}$ spectrum of W43-MM1 shows detections of the expected transitions mixed up with additional lines that we have identified (see Fig. 4). First, emission line from $\mathrm{H}_{2}^{18} \mathrm{O}$ is blended

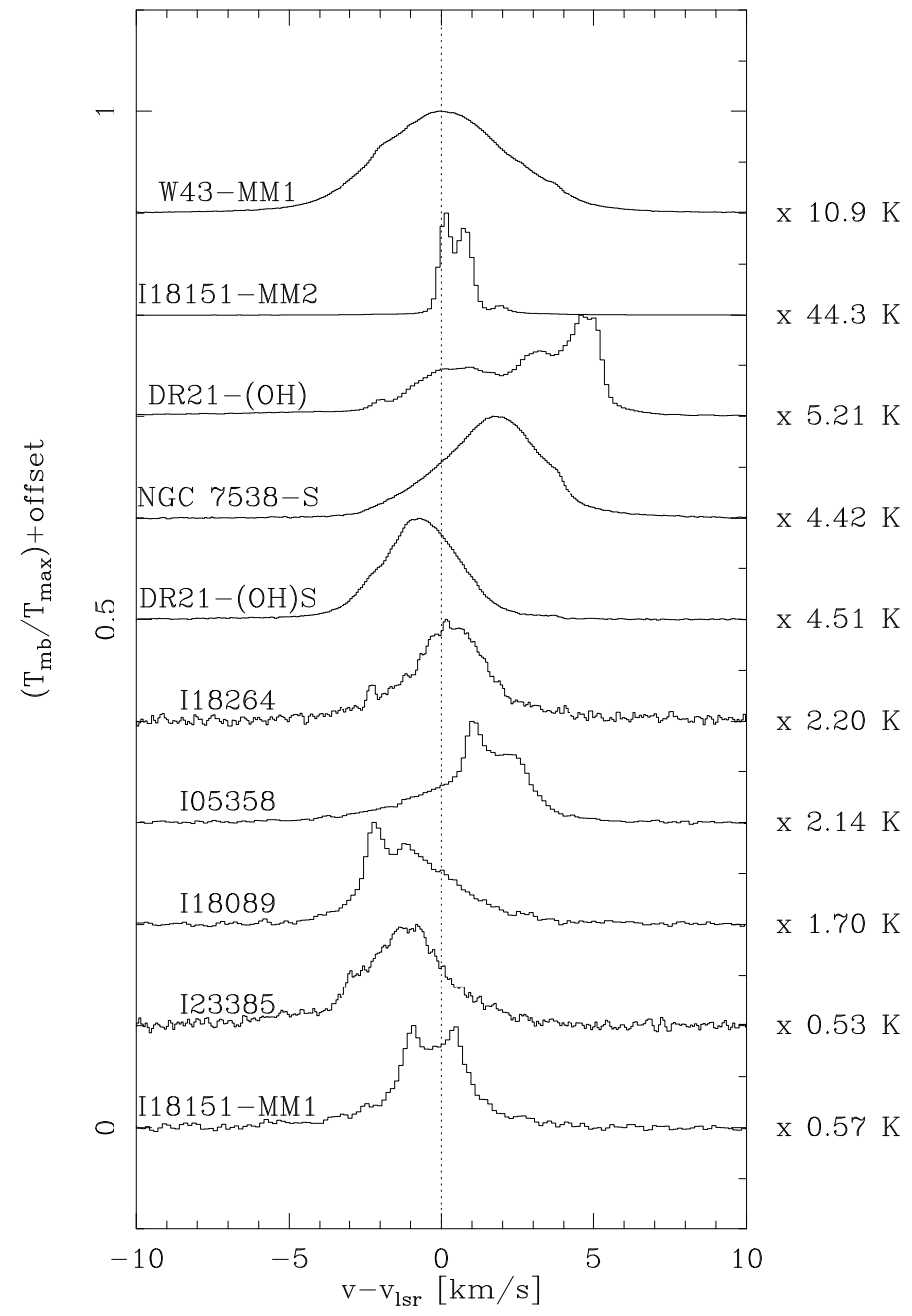

Fig. 1. Line profiles of the $\mathrm{CH}_{3} \mathrm{OH}$ emission observed in our sample. Profiles have been offset by $v_{\mathrm{lsr}}$ (see Table 1) and normalized to unity with a factor indicated on the right part of the panel.

with an emission line of the $\mathrm{CH}_{3} \mathrm{OCH}_{3}$ molecule at $203.408 \mathrm{GHz}$ (see Table 4). We also detect a mixing between the $\mathrm{SO}_{2}$ transition and emission at the frequency of $203.392 \mathrm{GHz}$ (maybe $\mathrm{C}_{3} \mathrm{H}_{7} \mathrm{CN}$, as discovered by Belloche et al. 2009). This emission seems to be real, as supported by its velocity width that is similar to other lines (see Table 4). In the same spectrum, three other emission lines from $\mathrm{CH}_{3} \mathrm{OCH}_{3}$ are clearly detected. All the lines have a Gaussian shape. The $3 \mathrm{~mm}$ spectrum of W43MM1 shows an unexpected feature near the $\mathrm{CH}_{3} \mathrm{OH}$ line, coming from the $\mathrm{NH}_{2} \mathrm{CHO}$ molecule (see Table 4). One also notes in IRAS 18089-1732 and DR21(OH) that the same group of 

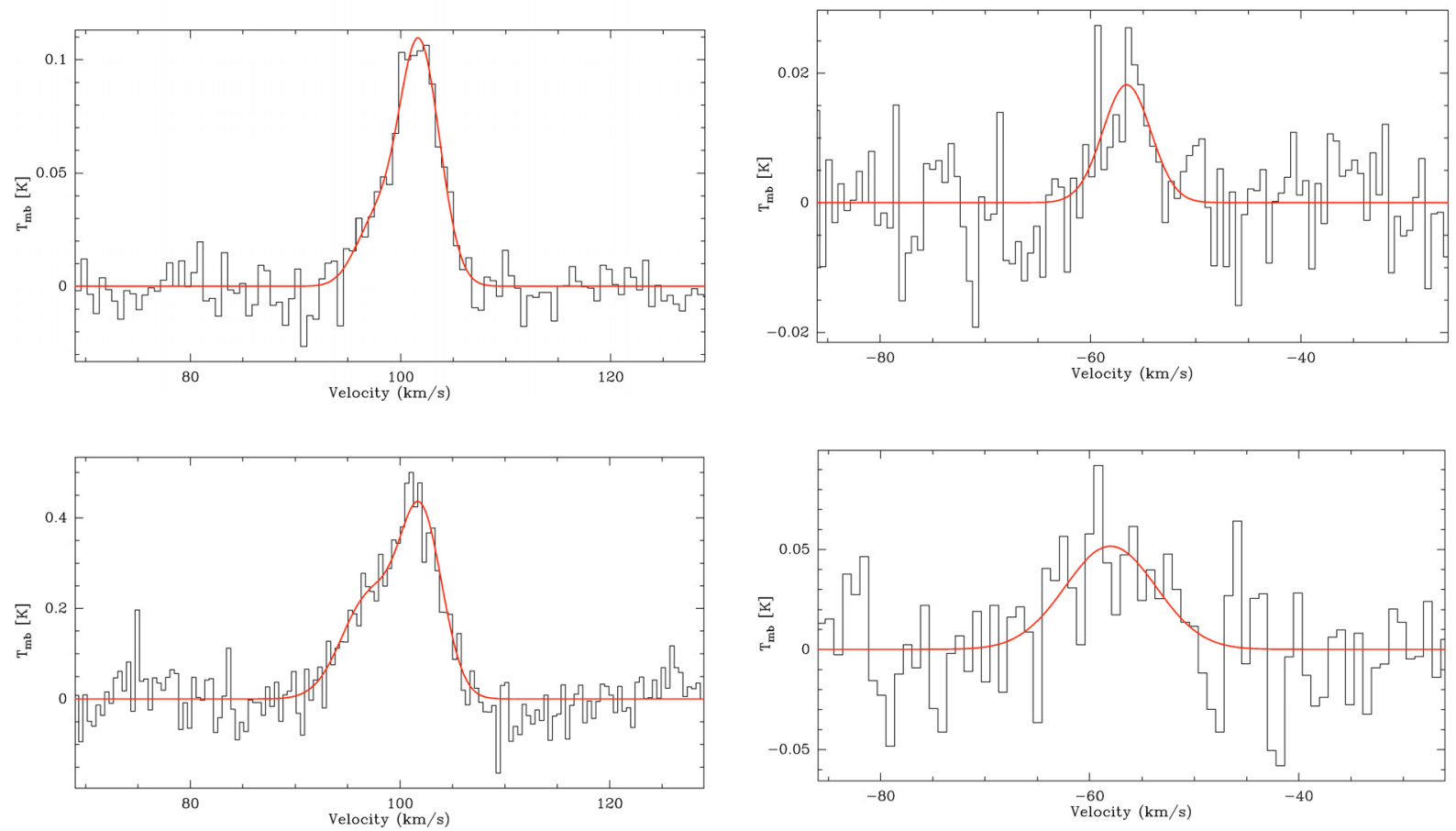

Fig. 2. Observations of the HDO (1-1) (top) and HDO (3-2) (bottom) emission lines from W43-MM1 (left) and NGC 7538S (right). For a clearer view, spectra have been smoothed by reducing the velocity resolution by a factor 2 (see Table 3 for the initial value).
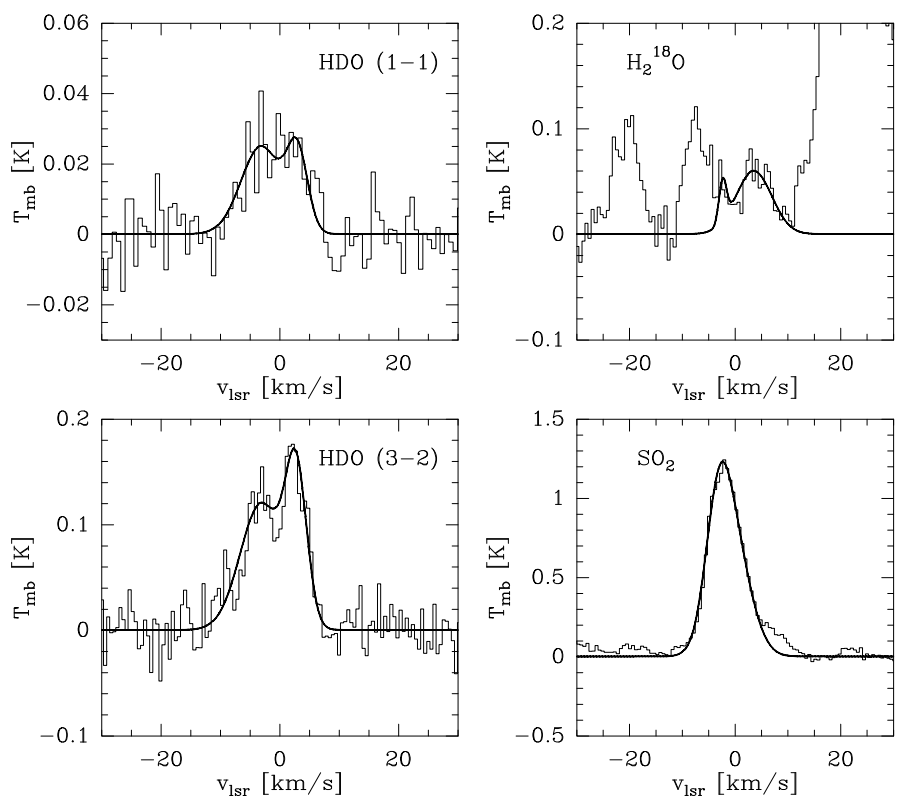

Fig. 3. Line profiles of the HDO at $3 \mathrm{~mm}$ (top left), $1 \mathrm{~mm}$ (bottom left), $\mathrm{H}_{2}^{18} \mathrm{O}$ (top right), and $\mathrm{SO}_{2}$ (bottom right) observed in DR21(OH) with their best double-Gaussian fit. Double component fit of $\mathrm{H}_{2}^{18} \mathrm{O}$ must be considered as tentative to be consistent with HDO line profiles. Profiles are smoothed by a factor of 5 for a better view.

$\mathrm{CH}_{3} \mathrm{OCH}_{3}$ lines seen in the W43-MM1 spectrum are detected. One of them is blended with $\mathrm{H}_{2}^{18} \mathrm{O}$.

\subsection{Line profiles}

For all sources, except W43-MM1, the methanol emission line profiles exhibit wings (Fig. 1). Some of them are seen in both

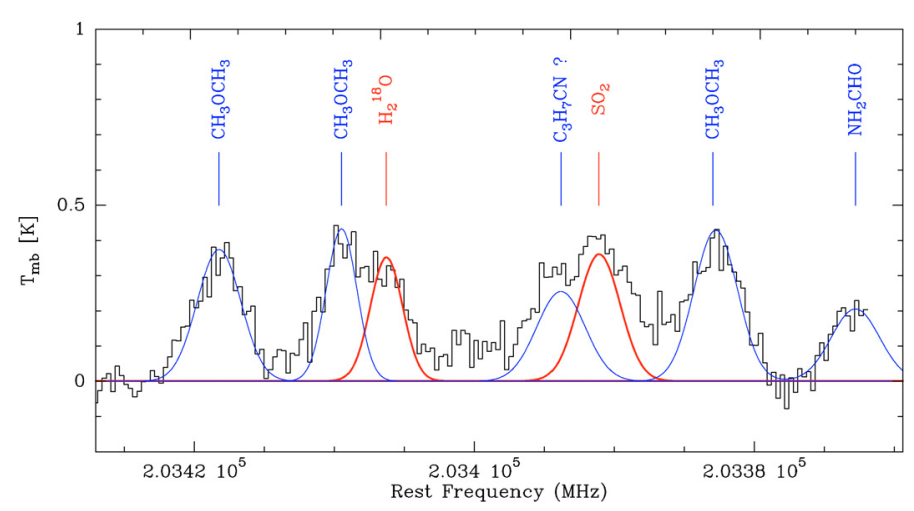

Fig. 4. Spectrum of $\mathrm{H}_{2}^{18} \mathrm{O}$ and $\mathrm{SO}_{2}$ emission lines from W43-MM1, showing the serendipitous detection of $\mathrm{CH}_{3} \mathrm{OCH}_{3}, \mathrm{C}_{3} \mathrm{H}_{7} \mathrm{CN}$ and $\mathrm{NH}_{2} \mathrm{CHO}$ lines. Spectra are smoothed by reducing the velocity resolution by a factor 8 (see Table 3 for the initial value).

the blue and red-shifted parts (IRAS 05358+3543, IRAS 181511208 1, IRAS 18151-1208 2, NGC 7538S), and others only on one side (blue-shifted for IRAS $23385+6053$, red-shifted for IRAS 18264-1152 and DR21(OH)S).

In addition to their wings, the methanol lines often show multiple velocity components. This is seen in IRAS 05358+3543, IRAS 18089-1732, IRAS 18151-1208MM2, W43-MM1, and DR21(OH). The number of these components varies between 3 and 4 and their strength from $\sim 1$ to $40 \mathrm{~K}$.

In DR21(OH), the $1.3 \mathrm{~mm}$ HDO transition exhibits a well defined two-component velocity pattern. A double Gaussian fit reproduces the observed line with the parameters reported in Table 5. The $3 \mathrm{~mm}$ HDO spectrum does not show the two velocity peaks seen at $1.3 \mathrm{~mm}$. A single velocity Gaussian profile provides a good fit but only with a rather large $\left(>10 \mathrm{~km} \mathrm{~s}^{-1}\right)$ line 
Early evolutionary stages of high-mass star formation: M. G. Marseille et al.

Table 3. Characteristics of observed lines.

\begin{tabular}{|c|c|c|c|c|c|c|c|}
\hline Source & Species & Transition & $\begin{array}{c}v_{\mathrm{lsr}} \\
\left(\mathrm{km} \mathrm{s}^{-1}\right)\end{array}$ & $\begin{array}{l}T_{\mathrm{mb}}{ }^{a} \\
(\mathrm{mK})\end{array}$ & $\begin{array}{c}\Delta v^{b} \\
\left(\mathrm{~km} \mathrm{~s}^{-1}\right)\end{array}$ & $\underset{\left(\mathrm{K} \mathrm{km} \mathrm{s}^{-1}\right)}{\int T d v}$ & $\begin{array}{l}\sigma_{\mathrm{rms}} \\
(\mathrm{mK})\end{array}$ \\
\hline \multirow[t]{4}{*}{ IRAS $05358+3543$} & HDO & $J=1_{1,0}-1_{0,1}$ & $\ldots$ & .. & $\ldots$ & $\ldots$ & 20 \\
\hline & HDO & $J=3_{2,1}-2_{2,1}$ & $\ldots$ & $\ldots$ & $\ldots$ & $\ldots$ & 40 \\
\hline & $\mathrm{H}_{2}^{18} \mathrm{O}$ & $J=3_{1,3}-2_{2,0}$ & $\ldots$ & $\ldots$ & $\ldots$ & $\ldots$ & 30 \\
\hline & $\mathrm{SO}_{2}$ & $J=12_{0,12}-11_{1,11}$ & $-15.9(4)$ & 90 & $6.6(5)$ & $0.61(4)$ & 30 \\
\hline \multirow[t]{4}{*}{ IRAS 18089-1732 } & HDO & $J=1_{1,0}-1_{0,1}$ & $32.1(1)$ & 130 & $4.5(3)$ & $0.62(3)$ & 10 \\
\hline & $\mathrm{HDO}$ & $J=3_{2,1}-2_{2,1}$ & $32.7(1)$ & 420 & $6.3(3)$ & $2.8(1)$ & 50 \\
\hline & $\mathrm{H}_{2}^{18} \mathrm{O}$ & $J=3_{1,3}-2_{2,0}$ & $32.7(2)$ & 180 & $5.7(2)$ & $1.09(7)$ & 30 \\
\hline & $\mathrm{SO}_{2}$ & $J=12_{0,12}-11_{1,11}$ & $32.9(4)$ & 430 & $9.5(4)$ & $4.4(1)$ & 30 \\
\hline \multirow[t]{4}{*}{ IRAS 18151-1208-MM1 } & HDO & $J=1_{1,0}-1_{0,1}$ & $\ldots$ & $\ldots$ & $\ldots$ & $\ldots$ & 20 \\
\hline & HDO & $J=3_{2,1}-2_{2,1}$ & $\ldots$ & .. & $\ldots$ & $\ldots$ & 50 \\
\hline & $\mathrm{H}_{2}^{18} \mathrm{O}$ & $J=3_{1,3}-2_{2,0}$ & $\ldots$ & $\ldots$ & $\ldots$ & .. & 40 \\
\hline & $\mathrm{SO}_{2}$ & $J=12_{0,12}-11_{1,11}$ & $33.3(3)$ & 80 & $4.2(8)$ & $0.36(5)$ & 40 \\
\hline \multirow[t]{4}{*}{ IRAS 18151-1208-MM2 } & HDO & $J=1_{1,0}-1_{0,1}$ & $\ldots$ & $\ldots$ & $\ldots$ & $\ldots$ & 20 \\
\hline & HDO & $J=3_{2,1}-2_{2,1}$ & $\ldots$ & .. & $\ldots$ & $\ldots$ & 60 \\
\hline & $\mathrm{H}_{2}^{18} \mathrm{O}$ & $J=3_{1,3}-2_{2,0}$ & $\ldots$ & $\ldots$ & $\ldots$ & .. & 50 \\
\hline & $\mathrm{SO}_{2}$ & $J=12_{0,12}-11_{1,11}$ & $\ldots$ & $\ldots$ & $\ldots$ & $\ldots$ & 50 \\
\hline \multirow[t]{4}{*}{ IRAS $18264-1152$} & HDO & $J=1_{1,0}-1_{0,1}$ & $\ldots$ & $\ldots$ & $\ldots$ & .. & 60 \\
\hline & HDO & $J=3_{2,1}-2_{2,1}$ & $\ldots$ & $\ldots$ & $\ldots$ & $\ldots$ & 330 \\
\hline & $\mathrm{H}_{2}^{18} \mathrm{O}$ & $J=3_{1,3}-2_{2,0}$ & $\ldots$ & $\ldots$ & .. & $\ldots$ & 290 \\
\hline & $\mathrm{SO}_{2}$ & $J=12_{0,12}-11_{1,11}$ & $\ldots$ & $\ldots$ & $\ldots$ & $\ldots$ & 290 \\
\hline \multirow[t]{4}{*}{ W43-MM1 } & $\mathrm{HDO}$ & $J=1_{1,0}-1_{0,1}$ & 101.3(1) & 107 & $5.7(2)$ & $0.65(2)$ & 20 \\
\hline & $\mathrm{HDO}$ & $J=3_{2,1}-2_{2,1}$ & $100.4(1)$ & 410 & $7.5(2)$ & $3.24(9)$ & 60 \\
\hline & $\mathrm{H}_{2}^{18} \mathrm{O}$ & $J=3_{1,3}-2_{2,0}$ & $100.6(5)$ & 220 & $3.8(7)$ & $1.1(3)$ & 80 \\
\hline & $\mathrm{SO}_{2}$ & $J=12_{0,12}-11_{1,11}$ & $100.7(3)$ & 230 & $5.2(7)$ & $1.29(6)$ & 80 \\
\hline \multirow[t]{4}{*}{ DR21(OH)-S } & HDO & $J=1_{1,0}-1_{0,1}$ & $\ldots$ & $\ldots$ & $\ldots$ & $\ldots$ & 20 \\
\hline & HDO & $J=3_{2,1}-2_{2,1}$ & $\ldots$ & .. & $\ldots$ & $\ldots$ & 30 \\
\hline & $\mathrm{H}_{2}^{18} \mathrm{O}$ & $J=3_{1,3}-2,0$ & $\ldots$ & $\ldots$ & $\ldots$ & .. & 40 \\
\hline & $\mathrm{SO}_{2}$ & $J=12_{0,12}-11_{1,11}$ & $-3.5(1)$ & 240 & $3.5(2)$ & $0.87(3)$ & 10 \\
\hline \multirow[t]{4}{*}{ NGC 7538S } & HDO & $J=1_{1,0}-1_{0,1}$ & $-56.7(5)$ & 20 & $6(1)$ & $0.11(2)$ & 20 \\
\hline & $\mathrm{HDO}$ & $J=3_{2,1}-2_{2,1}$ & $-57.9(8)$ & 20 & $10(2)$ & $0.57(9)$ & 50 \\
\hline & $\mathrm{H}_{2}^{18} \mathrm{O}$ & $J=3_{1,3}-2_{2,0}$ & $\ldots$ & .. & $\ldots$ & $\ldots$ & 50 \\
\hline & $\mathrm{SO}_{2}$ & $J=12_{0,12}-11_{1,11}$ & $-55.7(1)$ & 580 & $6.1(1)$ & $3.71(5)$ & 50 \\
\hline \multirow[t]{4}{*}{ IRAS $23385+6053$} & HDO & $J=1_{1,0}-1_{0,1}$ & $\ldots$ & $\ldots$ & $\ldots$ & ... & 10 \\
\hline & $\mathrm{HDO}$ & $J=3_{2,1}-2_{2,1}$ & $\ldots$ & $\ldots$ & $\ldots$ & .. & 60 \\
\hline & $\mathrm{H}_{2}^{18} \mathrm{O}$ & $J=3_{1,3}-2_{2,0}$ & .. & $\ldots$ & $\ldots$ & $\ldots$ & 30 \\
\hline & $\mathrm{SO}_{2}$ & $J=12_{0,12}-11_{1,11}$ & $-50.3(6)$ & 40 & $8(2)$ & $0.35(5)$ & 10 \\
\hline
\end{tabular}

Notes. Lines are fitted with a single Gaussian profile. Numbers in brackets indicates the error bar associated with the last decimal written. DR21(OH) is not reported here, having its own dedicated table. ${ }^{(a)}$ Conversion factor is $S / T_{\mathrm{mb}}=4.95 \mathrm{Jy} / \mathrm{K}$ for IRAM $30 \mathrm{~m}$ telescope. ${ }^{(b)}$ The line emission velocity width is measured at the full width half maximum (FWHM).

width. We find that a more convincing approach is to analyse this line with a two-line Gaussian model using fixed velocities and widths derived from the $1.3 \mathrm{~mm}$ line, obtaining a good result. The fit parameters are reported in Table 5. In the $\mathrm{H}_{2}^{18} \mathrm{O}$ spectrum, dimethyl ether and $\mathrm{SO}_{2}$ lines are clearly detected. A close examination of all dimethyl ether lines reveals that not all lines are well fitted with a single velocity component model because lines are too wide compared to $1.3 \mathrm{~mm}$ HDO components. We choose to fit those lines with a two-component model derived from the peak velocities observed in the methanol spectrum. We can then remove from the spectrum the dimethyl ether contribution. The resulting spectrum shows a signal well above the noise in the range expected for the $\mathrm{H}_{2}^{18} \mathrm{O}$ transition. The higher velocity part is around the $2.9 \mathrm{~km} \mathrm{~s}^{-1}$ velocity observed in the $1.3 \mathrm{~mm}$ HDO spectrum and is well above the noise. It is also well separated from the dimethyl ether line (no blending). On the other hand, the lower velocity part, around the $-3.2 \mathrm{~km} \mathrm{~s}^{-1}$ component of the HDO line is partly blended by dimethyl ether and the signal-tonoise ratio is lower. Fit results are given in Table 5.
To estimate the $\mathrm{H}_{2}^{18} \mathrm{O}$ emission line strength, and be consistent with the observed HDO line profiles, the $\mathrm{CH}_{3} \mathrm{OCH}_{3}$ lines were first subtracted from the spectrum using a model with two velocity components. The $\mathrm{CH}_{3} \mathrm{OCH}_{3}$ and water emissions are expected to originate in the same region because these two species are chemically linked. We then tentatively applied the velocity component values to fit the $\mathrm{H}_{2}^{18} \mathrm{O}$ emission. The resulting line emission parameters are reported in Table 5, showing a blue-shifted component that remains doubtful, even if it cannot be completely rejected (see Fig. 3). The $\mathrm{SO}_{2}$ line is detected but a single velocity component fit does not provide a satisfying profile, where a two-velocity fit constrained by the velocity difference (taken from the methanol velocities) gives a slightly better result (see Table 5). The remaining red wing on the $\mathrm{SO}_{2}$ line is mainly related to the dimethyl ether line. The remaining blue signal on the foot of $\mathrm{SO}_{2}$ cannot be identified, but is weak $(70 \mathrm{mK})$. 
Table 4. Characteristics of the unexpected emission lines detected in W43-MM1.

\begin{tabular}{lccccc}
\hline \hline Species & $\begin{array}{c}\text { Frequency } \\
(\mathrm{GHz})\end{array}$ & $\begin{array}{c}E_{\mathrm{up}} \\
(\mathrm{K})\end{array}$ & $\begin{array}{c}T_{\mathrm{mb}} \\
(\mathrm{mK})\end{array}$ & $\begin{array}{c}\Delta v^{b} \\
\left(\mathrm{~km} \mathrm{~s}^{-1}\right)\end{array}$ & $\begin{array}{c}\int T \mathrm{~d} v \\
\left(\mathrm{~K} \mathrm{~km} \mathrm{~s}^{-1}\right)\end{array}$ \\
\hline $\mathrm{NH}_{2} \mathrm{CHO}$ & 84.542 & 7 & 190 & $6.51(9)$ & $1.35(2)$ \\
$\mathrm{CH}_{3} \mathrm{OCH}_{3}$ & 203.383 & 9 & 430 & $5.3(5)$ & $2.5(3)$ \\
$\mathrm{CH}_{3} \mathrm{OCH}_{3}$ & 203.406 & 113 & 430 & $3.8(7)$ & $1.8(2)$ \\
$\mathrm{CH}_{3} \mathrm{OCH}_{3}$ & 203.418 & 9 & 370 & $5.3(4)$ & $2.2(3)$ \\
$\mathrm{CH}_{3} \mathrm{CHO}^{a}$ & 211.936 & 444 & 260 & $6(1)$ & $1.6(3)$ \\
$\mathrm{CH}_{3} \mathrm{CHO}$ & 211.957 & 216 & 210 & $6(2)$ & $1.3(4)$ \\
\hline
\end{tabular}

Notes. Numbers in brackets indicate the error bar associated to the last decimal written. ${ }^{(a)}$ Uncertain assignment due to the high level of the upper energy. It could be $\mathrm{C}_{3} \mathrm{H}_{7} \mathrm{CN}$, as discovered by Belloche et al. (2009) or a $\mathrm{SO}_{2}$ extension of the main emission at $\Delta v=-6.2 \mathrm{~km} \mathrm{~s}^{-1}$ induced by outflows. ${ }^{(b)}$ The line emissionvelocity width is measured at the FHWM.

Table 5. Components in the line profile of DR21(OH).

\begin{tabular}{llcccc}
\hline \hline Species & Transition & Component & $\begin{array}{c}v \\
\left(\mathrm{~km} \mathrm{~s}^{-1}\right)\end{array}$ & $\begin{array}{c}T_{\mathrm{mb}} \\
(\mathrm{mK})\end{array}$ & $\begin{array}{c}\Delta v^{a} \\
\left(\mathrm{~km} \mathrm{~s}^{-1}\right)\end{array}$ \\
\hline $\mathrm{HDO}$ & $J=1_{1,0}-1_{0,1}$ & 1 & $-3.2(4)$ & 120 & $8(1)$ \\
& $J=1_{1,0}-1_{0,1}$ & 2 & $2.9(1)$ & 140 & $4.2(3)$ \\
\hline $\mathrm{HDO}$ & $J=3_{2,1}-2_{2,1}$ & 1 & $-3.2(1)$ & 25 & $8(1)$ \\
& $J=3_{2,1}-2_{2,1}$ & 2 & $2.9(4)$ & 22 & $4.2(3)$ \\
\hline $\mathrm{H}_{2}^{18} \mathrm{O}$ & $J=3_{1,3}-2_{2,0}$ & 1 & $-2.4(2)$ & 40 & $1.7(6)$ \\
& $J=3_{1,3}-2_{2,0}$ & 2 & $3.5(1)$ & 60 & $8(1)$ \\
\hline $\mathrm{SO}_{2}$ & $J=12_{0,12}-11_{1,11}$ & 1 & $-3.3(1)$ & 640 & $5.6(1)$ \\
& $J=12_{0,12}-11_{1,11}$ & 2 & $-0.7(1)$ & 720 & $8.0(1)$ \\
\hline
\end{tabular}

Notes. Table presents HDO line components at $3 \mathrm{~mm}$ (bottom), $1.3 \mathrm{~mm}$ (top), $\mathrm{H}_{2}^{18} \mathrm{O}$, and $\mathrm{SO}_{2}$ in DR21(OH). Numbers in brackets indicates the error bar associated with the last decimal written. ${ }^{(a)}$ The line emission velocity width is measured at the FWHM. Values given for the $\mathrm{H}_{2}^{18} \mathrm{O}$ blue-shifted component are extracted for the best fit Gaussian function, which remains quite uncertain.

\section{Methanol maser emission}

The $\mathrm{CH}_{3} \mathrm{OH}\left(J=5_{-1,5}-4_{0,4} \mathrm{E}\right)$ transition is often detected as a so-called class I maser. Unlike class II masers, which originate in the immediate protostellar environment $\left(\lesssim 10^{3} \mathrm{AU}\right)$, class I masers arise at some distance $\left(\gtrsim 10^{4} \mathrm{AU}\right)$ from the protostar. Here, shocks between jets and ambient gas cause local density and temperature enhancements where population inversions may occur (e.g. Minier et al. 2005). As a consequence, the maser emission lines are very sensitive to the local environment in which they appear and typically observed to have sharply peaked profile that can be distinguished from thermal, turbulent, or outflow emissions.

In our observations, the line profiles show that the maser and thermal emissions are mixed in different proportions. As we aim to trace shocks, we need to extract maser emission to ensure a reliably determination of any relation between the supposed evolutionary stages of our sources and the strengths of the shocks that may occur inside. An identical analysis is performed with profiles obtained by van der Tak et al. (2006) to extend our study.

\subsection{Extraction method}

To disentangle the thermal and maser contributions to the $\mathrm{CH}_{3} \mathrm{OH}$ line profiles, we use the extraction method of Caswell et al. (2000). The strong turbulence within these sources is also relevant a role too, leading to a larger width of the Gaussian line profile. This is why we enclose its effect under the term "thermal" used in this paper. We fit the MDC's envelope contribution in each methanol profile by including the sources previously observed by van der Tak et al. (2006). The thermal emission is then fitted by a Gaussian component centred on the source velocity, an assumption motivated by all observations of class II or class I methanol masers (e.g. Caswell et al. 1995; Val'tts et al. 2000; Błaszkiewicz \& Kus 2004) exhibiting maser line profiles that are sharply peaked and differ from a Gaussian shape. If the line becomes saturated, the maser profile is of course no longer narrow and the wings grow exponentially (Elitzur 1992) but this will never produce a "thermally-looking" profile. The possibility of blends of emission originating in multiple maser spots within the telescope beam is also real, and our assumption cannot be definitely verified until future interferometer observations are made.

Once the maser emission has been extracted, we measure its velocity-integrated area $A_{\text {maser }}$. We also compute the area $A_{\text {therm }}$ of the thermal emission and derive the ratio $X_{\mathrm{m}}=A_{\text {maser }} / A_{\text {therm }}$ of these two quantities, to determine the sources in which the maser emission dominates.

\subsection{Results}

The results of the $\mathrm{CH}_{3} \mathrm{OH}$ class I maser emission extraction are given in Table 6, including results for the profiles observed by van der Tak et al. (2006).

The maser emission is dominant $\left(X_{\mathrm{m}}>10\right)$ in two sources, IRAS 18151-1208-MM2 and DR21(OH). The very high emission intensity in these objects $\left(T_{\text {peak }}=44.3\right.$ and $5.21 \mathrm{~K}$, respectively), and the structure of their profiles (see Fig. 1) are consistent with this finding. A group of four sources (IRAS 05358+3543, IRAS 18089-1732, NGC 7538S, and IRAS 23385+6053) exhibit distinctive maser emission, $\left(X_{\mathrm{m}} \sim 1-2\right)$. A majority of objects exhibit weak maser emission $\left(X_{\mathrm{m}} \sim 0.1-1.0\right)$ : IRAS 18151-1208-MM1, IRAS 18264-1152, W43-MM1, DR21(OH)-S, W33A, AFGL 2591, S140 IRS1, NGC 7538 IRS1, and NGC 7538 IRS9. In the three remaining objects, W3 IRS5, AFGL 2136, and AFGL 490, the maser emission seems to have disappeared and our measurements must be treated as upper limits (see Table 6).

\section{Molecular abundances}

\subsection{Modelling method}

To derive molecular abundances from our observations, we use a global modelling process developed by van der Tak et al. (1999) and improved by Marseille et al. (2008). We first model the SED with the MC3D program (Wolf et al. 1999) to derive the mass and the temperature distributions inside the MDCs. The source model, i.e., its total luminosity, size, and density distribution is constrained by observations (see Tables 1 and 7), and uses dust properties derived by Draine \& Lee (1984). We then transfer the density and temperature distribution to the RATRAN code (Hogerheijde \& van der Tak 2000). This non-LTE radiative transfer code models the molecular line emissions observed, and thus we obtain the molecular abundances and the turbulence level, which are the two free parameters of this method. 
Early evolutionary stages of high-mass star formation: M. G. Marseille et al.

Table 6. Results of the $\mathrm{CH}_{3} \mathrm{OH}$ class I maser emission extraction.

\begin{tabular}{|c|c|c|c|c|c|c|c|c|}
\hline \multirow[b]{2}{*}{ \# } & \multirow[b]{2}{*}{ Source } & \multicolumn{3}{|c|}{ Thermal } & \multicolumn{3}{|c|}{ Maser } & \multirow[b]{2}{*}{$X_{\mathrm{m}}$} \\
\hline & & $\begin{array}{l}T_{\text {th }} \\
(\mathrm{K})\end{array}$ & $\begin{array}{c}\Delta v \\
\left(\mathrm{~km} \mathrm{~s}^{-1}\right)\end{array}$ & $\begin{array}{c}A_{\mathrm{th}} \\
\left(\mathrm{K} \mathrm{km} \mathrm{s}^{-1}\right)\end{array}$ & $\begin{array}{l}T_{\mathrm{m}} \\
(\mathrm{K})\end{array}$ & $\begin{array}{c}v_{\mathrm{m}} \\
\left(\mathrm{km} \mathrm{s}^{-1}\right)\end{array}$ & $\begin{array}{c}A_{\mathrm{m}} \\
\left(\mathrm{Kkm} \mathrm{s}^{-1}\right)\end{array}$ & \\
\hline 1 & IRAS $05358+3543$ & 0.48 & 5.0 & 2.55 & $1.7 / 1.2$ & $-16.5 /-15.2$ & 3.94 & 1.54 \\
\hline 2 & IRAS 18089-1732 & 0.59 & 3.6 & 2.26 & $1.5 / 0.9$ & $31.6 / 32.6$ & 3.20 & 1.42 \\
\hline 3 & IRAS 18151-1208-MM1 & 0.44 & 2.2 & 1.03 & $0.3 / 0.2 / 0.1$ & $32.4 / 33.9 / 31.0$ & 0.72 & 0.70 \\
\hline 4 & IRAS 18151-1208-MM2 & 0.70 & 5.0 & 3.72 & $43.8 / 37.4 / 3.8$ & $29.9 / 30.5 / 31.6$ & 639.5 & 172 \\
\hline 5 & IRAS 18264-1152 & 1.20 & 3.8 & 4.85 & $1.0 / 0.4$ & $43.8 / 41.3$ & 2.68 & 0.55 \\
\hline 6 & W43-MM1 & 10.9 & 4.2 & 53.9 & $1.5 / 1.2$ & $102.0 / 96.8$ & 9.53 & 0.18 \\
\hline 7 & $\mathrm{DR} 21(\mathrm{OH})$ & 1.94 & 2.2 & 4.84 & $5.1 / 3.1 / 1.5 / 0.4$ & $0.9 /-1.2 /-3.1 /-6.5$ & 537.35 & 64 \\
\hline 8 & DR21(OH)-S & 3.50 & 2.2 & 8.19 & 2.4/1.8/0.2 & $-4.3 /-5.2 /-0.8$ & 6.78 & 0.83 \\
\hline 9 & NGC 7538S & 1.81 & 3.1 & 5.94 & $2.8 / 2.2$ & $-55.1 /-53.3$ & 13.2 & 2.22 \\
\hline 10 & IRAS $23385+6053$ & 0.25 & 2.6 & 0.79 & $0.4 / 0.3$ & $-50.3 /-51.8$ & 1.29 & 1.86 \\
\hline $\mathrm{a}$ & W3 IRS5 & 0.07 & 2.6 & 0.18 & $\ldots^{a}$ & $\ldots$ & $<5.4 \times 10^{-3}$ & $<0.03$ \\
\hline $\mathrm{b}$ & AFGL 490 & 0.14 & 2.4 & 0.36 &.$^{a}$ & $\ldots$ & $<3.6 \times 10^{-2}$ & $<0.10$ \\
\hline $\mathrm{c}$ & W33A & 1.51 & 3.5 & 5.59 & $0.4 / 0.2$ & $35.2 / 39.5$ & 2.74 & 0.49 \\
\hline $\mathrm{d}$ & AFGL 2136 & 0.17 & 2.9 & 0.52 & $\ldots^{a}$ & $\ldots$ & $<6 \times 10^{-4}$ & $<0.0012$ \\
\hline $\mathrm{e}$ & AFGL 2591 & 0.39 & 2.9 & 1.19 & $0.2 / 0.1$ & $-5.9 /-8.6$ & 0.46 & 0.38 \\
\hline $\mathrm{f}$ & S140 IRS1 & 0.43 & 2.1 & 0.95 & $0.2 / 0.1$ & $-5.7 /-8.2$ & 0.55 & 0.57 \\
\hline $\mathrm{g}$ & NGC 7538 IRS1 & 0.68 & 2.9 & 1.85 & 0.3 & -56.2 & 0.51 & 0.27 \\
\hline $\mathrm{h}$ & NGC 7538 IRS9 & 0.64 & 2.0 & 1.35 & $0.4 / 0.2$ & $-56.3 /-55.5$ & 1.00 & 0.74 \\
\hline
\end{tabular}

Notes. The table presents parameters of the main thermal emission fitted by a Gaussian (temperatures $T_{\text {th }}$, velocity widths at FHWM $\Delta v$ and area $A_{\text {therm }}$ ), the resulting maser emission peaks characteristics (temperatures $T_{\mathrm{m}}$ and velocities $v_{\mathrm{m}}$ ), and the area ratios $\left(X_{\mathrm{m}}\right)$. ${ }^{(a)}$ No methanol maser features detected over the $3 \sigma_{\text {rms }}$ noise threshold.

The density distribution is characterized by a power law of the form

$n(r)=n_{0}\left(\frac{r}{r_{0}}\right)^{-p}$

where $n_{0}$ is the density at the reference radius $r_{0}$ (here $100 \mathrm{AU}$ ) obtained by a fit to the optically thin emission of dust at millimetre wavelengths. The parameter $p$ is derived from continuum maps of MDCs or is set to be 1.5 according to a quasi-static infall theory in the inner part of the object (Shu et al. 1987; Beuther et al. 2002c, see Table 7). The density and temperature distributions are transferred to RATRAN when modelling the emission lines. The best fit is obtained for a given gas turbulence velocity $v_{\mathrm{T}}$ and a molecular abundance relative to $\mathrm{H}_{2}\left(X_{\mathrm{mol}}=n_{\mathrm{mol}} / n_{\mathrm{H}_{2}}\right)$. When an emission line is not detected, we derive an upper limit assuming a line velocity width similar to the $\mathrm{SO}_{2}$ one (if available, otherwise we select the thermal component of the methanol line) and refer to the abundance required for a $2 \sigma_{\text {rms }}$ signal.

\subsection{Results}

The HDO abundances obtained are between $10^{-13}$ and $10^{-9}$ when the line emission is detected (IRAS 18089-1732, W43-MM1, DR21(OH), and NGC 7538S; see Table 8). For other sources, the upper limits are between $10^{-13}$ and $10^{-11}$. IRAS $23385+6053$ is an exception with the high upper limit of $1.5 \times 10^{-9}$ for the HDO $\left(3_{1,2}-2_{2,1}\right)$ transition. This high value (see Table 8 ) is mainly due to the compact shape of the source $(0.05 \mathrm{pc}$ compared with the size of the other sources, i.e. $\sim 0.13 \mathrm{pc}$ ) that increases the beam dilution for high-excitation lines. Furthermore, IRAS $23385+6053$ is a very dense and hot source (see Table 7) where a high abundance of water species is needed to reach the level of the critical density, permitting us to avoid strong self-absorption of the line emission (particularly for high-energy transitions), and explaining our high upper limit.
When the emission line of $\mathrm{H}_{2}^{18} \mathrm{O}$ is detected (i.e. in IRAS 18089-1732, W43-MM1, and DR21(OH)), its abundance is inferred to be between $10^{-11}$ and $10^{-9}$, giving a $\mathrm{H}_{2}^{16} \mathrm{O}$ abundance estimation between $5 \times 10^{-8}$ and $5 \times 10^{-7}$, assuming a solar isotopic ratio ${ }^{16} \mathrm{O} /{ }^{18} \mathrm{O}=500$. Other sources give an upper limit between $10^{-13}$ and $10^{-12}$, hence a main water isotope abundance between $5 \times 10^{-11}$ and $5 \times 10^{-10}$. This result seems to indicate a low water abundance in the MDC concerned, relative to the values measured by van der Tak et al. (2006, see Tables 8 and 9), except for W43-MM1. The comparison of these abundances with those obtained with the high-energy transition of HDO $\left(E_{\text {up }}=168 \mathrm{~K}\right.$, hence close to the $\mathrm{H}_{2}^{18} \mathrm{O}$ transition considered) show that they are consistent and higher than the lowenergy transition of HDO ( $E_{\text {up }}=47 \mathrm{~K}$ ), except W43-MM1 (see Sect. 8 for a discussion of this result).

The $\mathrm{HDO}$ and $\mathrm{H}_{2}^{18} \mathrm{O}$ abundances that we derive permit us to estimate the $\mathrm{HDO} / \mathrm{H}_{2} \mathrm{O}$ ratio in MDCs. Assuming that ${ }^{16} \mathrm{O} /{ }^{18} \mathrm{O}=500$, the ratios are $33 \times 10^{-4}, 7 \times 10^{-4}$, and $19 \times 10^{-4}$ for IRAS 18089-1732, W43-MM1, and DR21(OH), respectively. These values are obtained from high-energy transition results (see Table 9). The $\mathrm{D} / \mathrm{H}$ ratio agrees with the values obtained by van der Tak et al. (2006), giving values between $3 \times 10^{-4}$ and $38 \times 10^{-4}$. Our results and previous ones are reported in Table 9 .

The $\mathrm{CH}_{3} \mathrm{OH}$ abundances are derived from the thermal emission components and are spread between $10^{-10}$ and $10^{-8}$, i.e. of the same order of magnitude as those derived by van der Tak et al. (2006) for mIRb MDCs. We note that W43-MM1 has the highest abundance in the sample, with a value reaching $5.4 \times 10^{-7}$.

The modelling of the $\mathrm{SO}_{2}$ emission line implies an abundance of between $10^{-12}$ and $10^{-10}$ (see Table 8), which confirms previous estimations in MDCs (van der Tak et al. 2003; Wakelam et al. 2004). In particular, W43-MM1, IRAS 05358+3543, and IRAS 18264-1152 were already studied in Herpin et al. (2009), where a multi-transition evaluation of abundances is 
Table 7. Modelling parameters.

\begin{tabular}{clcccccc}
\hline \hline$\#$ & Source & $\begin{array}{c}n_{0} \\
\left(\text { grains m}^{-3}\right)\end{array}$ & $p$ & $\begin{array}{c}\langle n\rangle \\
\left(\mathrm{cm}^{-3}\right)\end{array}$ & $\alpha$ & $\begin{array}{c}T_{0} \\
(\mathrm{~K})\end{array}$ & $\begin{array}{c}\langle T\rangle \\
(\mathrm{K})\end{array}$ \\
\hline 1 & IRAS 05358+3543 & $1.5 \times 10^{3}$ & 1.4 & $8.4 \times 10^{5}$ & -0.62 & 562 & 31.1 \\
2 & IRAS 18089-1732 & $7.4 \times 10^{4}$ & 1.5 & $6.1 \times 10^{7}$ & -0.46 & 407 & 36.1 \\
3 & IRAS 18151-1208-MM1 & $8.7 \times 10^{2}$ & 1.2 & $5.5 \times 10^{5}$ & -0.60 & 631 & 32.5 \\
4 & IRAS 18151-1208-MM2 & $7.5 \times 10^{2}$ & 1.3 & $6.5 \times 10^{5}$ & -0.61 & 427 & 24.2 \\
5 & IRAS 18264-1152 & $4.0 \times 10^{3}$ & 1.5 & $9.8 \times 10^{5}$ & -0.61 & 676 & 32.6 \\
6 & W43-MM1 & $2.0 \times 10^{5}$ & 2.0 & $5.5 \times 10^{6}$ & -0.54 & 302 & 20.0 \\
7 & DR21(OH) & $4.1 \times 10^{4}$ & 1.5 & $3.5 \times 10^{7}$ & -0.44 & 191 & 20.7 \\
8 & DR21(OH)-S & $1.8 \times 10^{4}$ & 1.5 & $1.5 \times 10^{7}$ & -0.43 & 224 & 25.3 \\
9 & NGC 7538S & $2.9 \times 10^{4}$ & 1.5 & $2.4 \times 10^{7}$ & -0.45 & 316 & 30.4 \\
10 & IRAS 23385+6053 & $5.4 \times 10^{5}$ & 1.5 & $5.0 \times 10^{8}$ & -0.42 & 347 & 38.7 \\
\hline
\end{tabular}

Notes. Table presents the source name, the number of dust grains $n_{0}$ per meter cube at $r_{0}=100$ AU (following a standard MRN distribution in size and types), the power-law index $p$, the mean $\mathrm{H}_{2}$ density in the source, the best-fit temperature distribution coefficients $\left(T=T_{0}\left(r / r_{0}\right)^{\alpha}\right)$ and the mean temperature in the source.

Table 8. Modelling results.

\begin{tabular}{|c|c|c|c|c|c|c|c|c|c|c|}
\hline \multirow{3}{*}{ Source } & \multirow{3}{*}{\multicolumn{3}{|c|}{$\begin{array}{l}X_{\mathrm{mol}} \\
\mathrm{H}_{2}^{18} \mathrm{O}\end{array}$}} & \multirow{3}{*}{$\mathrm{SO}_{2}$} & \multirow{3}{*}{$\mathrm{CH}_{3} \mathrm{OH}$} & \multicolumn{5}{|c|}{$2 v_{\mathrm{T}}\left(\mathrm{km} \mathrm{s}^{-1}\right)$} \\
\hline & & & & & & \multicolumn{2}{|c|}{ HDO } & \multirow{2}{*}{$\mathrm{H}_{2}^{18} \mathrm{O}$} & \multirow{2}{*}{$\mathrm{SO}_{2}$} & \multirow{2}{*}{$\mathrm{CH}_{3} \mathrm{OH}$} \\
\hline & & & & & & $3 \mathrm{~mm}$ & $1 \mathrm{~mm}$ & & & \\
\hline IRAS $05358+3543$ & $<3.8(-12)$ & $<7.6(-13)$ & $<4.8(-13)$ & $5.1(-11)$ & $1.3(-9)$ & 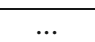 & 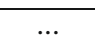 & ... & 2.4 & 2.9 \\
\hline IRAS 18089-1732 & $2.0(-11)$ & $1.3(-10)$ & $7.9(-11)$ & $4.2(-11)$ & $1.3(-10)$ & 2.4 & 4.2 & 5.2 & 5.8 & 2.1 \\
\hline IRAS 18151-1208-MM1 & $<5.8(-13)$ & $<2.2(-13)$ & $<5.2(-13)$ & $1.2(-10)$ & $1.6(-9)$ & $\ldots$ & $\ldots$ & $\ldots$ & 2.5 & 1.4 \\
\hline IRAS 18151-1208-MM2 & $<2.0(-12)$ & $<1.1(-12)$ & $<1.8(-12)$ & $<1.2(-10)$ & $1.0(-8)$ & $\ldots$ & $\ldots$ & $\ldots$ & $\ldots$ & 3.2 \\
\hline IRAS $18264-1152$ & $<2.3(-12)$ & $<1.6(-12)$ & $<1.1(-12)$ & $<1.2(-10)$ & $3.2(-9)$ & $\ldots$ & $\ldots$ & .. & $\ldots$ & 2.4 \\
\hline W43-MM1 & $1.5(-9)$ & $1.7(-9)$ & $4.6(-9)$ & $2.4(-10)$ & $4.8(-7)$ & 2.9 & 2.9 & 2.7 & 3.3 & 4.2 \\
\hline $\mathrm{DR} 21(\mathrm{OH})^{a}$ & $6.1(-13)$ & $8.0(-11)$ & $8.5(-11)$ & $8.4(-11)$ & $1.0(-10)$ & 2.3 & 3.2 & 1.7 & 3.7 & 1.1 \\
\hline DR21(OH)-S & $<8.0(-14)$ & $<2.0(-11)$ & $<1.8(-13)$ & $7.8(-12)$ & $4.0(-10)$ & $\ldots$ & $\ldots$ & .. & 2.0 & 1.2 \\
\hline NGC 7538S & $8.9(-14)$ & $1.3(-13)$ & $<2.8(-13)$ & $3.4(-11)$ & $4.5(-10)$ & 2.8 & 2.6 & $\ldots$ & 3.5 & 1.9 \\
\hline IRAS $23385+6053$ & $<1.5(-13)$ & $<1.5(-9)$ & $<1.5(-10)$ & $3.4(-11)$ & $9.4(-10)$ & $\ldots$ & $\ldots$ & $\ldots$ & 4.8 & 1.0 \\
\hline
\end{tabular}

Notes. Table presents molecular abundances relative to $\mathrm{H}_{2}$ and turbulent velocities derived from the global modelling method. Values are indicated with the form $x(-y)=x \times 10^{-y}$. When a line emission is not detected, the upper limit assume a line velocity width similar to the $\mathrm{SO}_{2}$ one and refers to the abundance required for a $2 \sigma_{\text {rms }}$ signal. ${ }^{(a)}$ Two velocity components with different line widths.

Table 9. Abundance ratios.

\begin{tabular}{llcc}
\hline \hline$\#$ & Source & $\begin{array}{c}\mathrm{HDO} / \mathrm{H}_{2} \mathrm{O} \\
\times 10^{-4}\end{array}$ & $\begin{array}{c}\mathrm{H}_{2} \mathrm{O} \\
\times 10^{-8}\end{array}$ \\
\hline 2 & IRAS 18089-1732 & 33 & 4.0 \\
6 & W43-MM1 & 7 & 230 \\
7 & DR21(OH) & 19 & 4.3 \\
\hline a & W3 IRS5 & 13 & 30 \\
c & W33A & 30 & 80 \\
e & AFGL 2591 & 3 & 40 \\
f & NGC 7538 IRS1 & 38 & 50 \\
\hline
\end{tabular}

Notes. Table presents $\mathrm{HDO} / \mathrm{H}_{2} \mathrm{O}$ ratios and water abundance derived from $\mathrm{H}_{2}^{18} \mathrm{O}$. Values in the bottom part are taken from radiative transfer model results and $\mathrm{H}_{2}^{18} \mathrm{O}$ column densities of van der Tak et al. (2006).

performed. For these sources, our results agree with their estimations. Even in IRAS 18264-1152, where an upper limit has been derived, our result is in accordance with the abundance estimated in their study.

We derive turbulent velocities higher than the speed of sound $\left(v_{\mathrm{T}}=0.85-2.9 \mathrm{~km} \mathrm{~s}^{-1}\right.$ compared to $\left.a_{\mathrm{s}}=0.3-0.5 \mathrm{~km} \mathrm{~s}^{-1}\right)$, confirming this MDC characteristic.

A large number of sources in our sample are found to have a low upper limit to their $\mathrm{H}_{2}^{18} \mathrm{O}$ abundances (see Table 8 ). We suspect that most of the water is frozen in a solid state on the surface on dust grains in these cases. As for HDO, IRAS 23385+6053 is an exception but this result can be easily explained (see second paragraph). At the same time, some sources have a clear water abundance enhancement: results on W43-MM1 confirm that it hosts a HMC (already detected by Motte et al. 2003; Herpin et al. 2009), and others on IRAS 18089-1732 and DR21(OH) may indicate the same.

\section{Correlations}

To investigate how the molecular line emission is related to the physical properties of the MDCs, we compare the line fluxes and abundances to the mid-IR $(12 \mu \mathrm{m})$ emission seen by MSX and IRAS. Previous studies identified a link between mid-infrared emission and the physical evolution of MDCs (van der Tak et al. 2000a; Marseille et al. 2008). To increase the sample size, we include the data collected by van der Tak et al. (2006) for eight mIRb-MDCs.

\subsection{Method}

We compute the correlation factors $\rho_{x, y}$ (see Appendix A) between the following parameters: mass $M$, luminosity $L$, thermal line width $\delta v$ (from the $\mathrm{SO}_{2}$ emission or, if not detected, thermal component of $\mathrm{CH}_{3} \mathrm{OH}$ ), monochromatic luminosity $L_{12}$ at $12 \mu \mathrm{m}$, the molecular emission-line flux observed in $\mathrm{CH}_{3} \mathrm{OH}$ (masered $A_{\mathrm{m}}$, thermal $A_{\mathrm{t}}$, the ratio $X_{\mathrm{m}}$, and the abundance $X_{\mathrm{mol}}$ ) and the abundance of $\mathrm{H}_{2} \mathrm{O}$ and $\mathrm{HDO}$ in sources where they are 
Early evolutionary stages of high-mass star formation: M. G. Marseille et al.
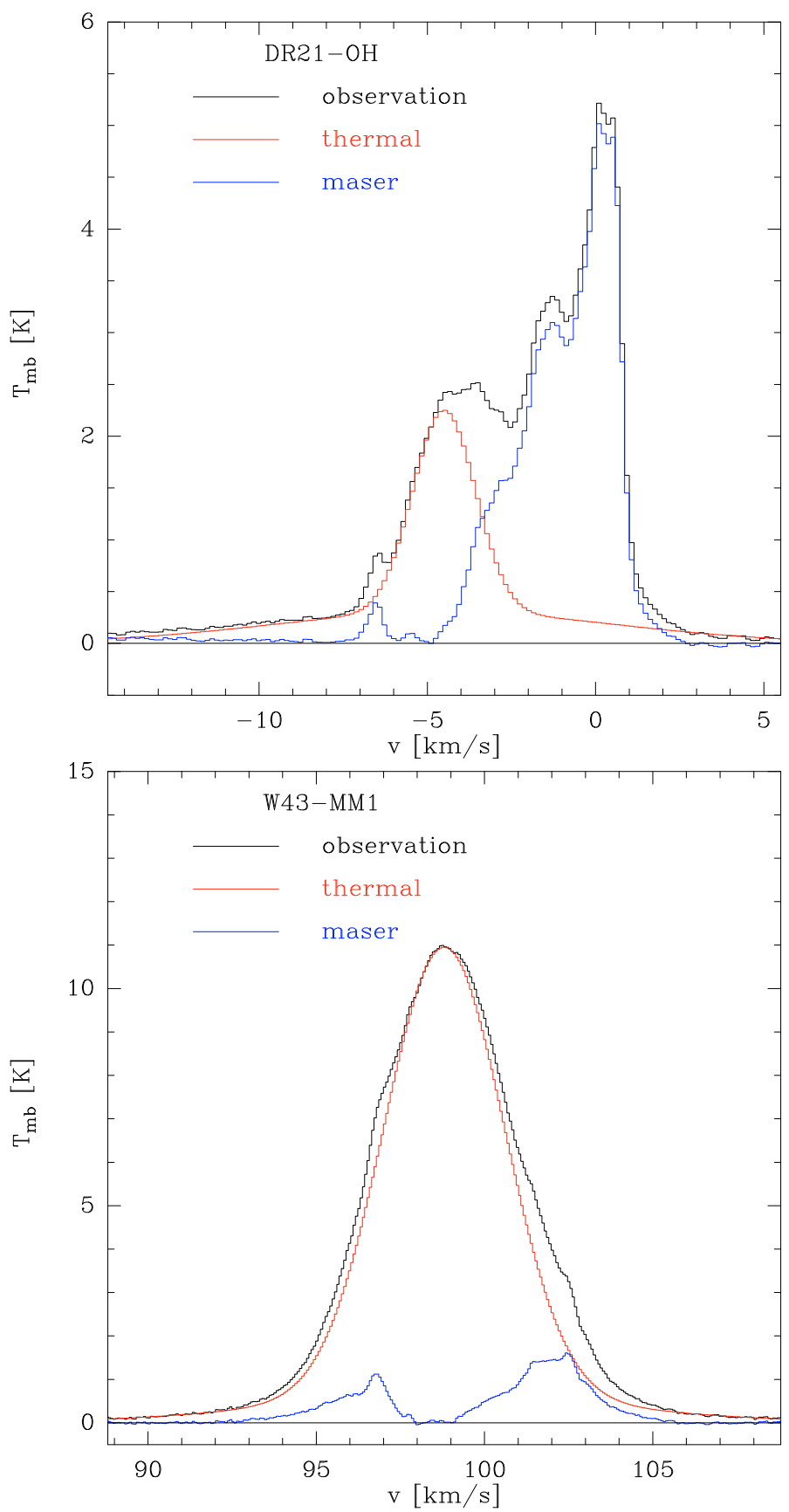

Fig. 5. Extraction of the methanol class I maser emission (blue line) from observations (black line) by fitting the thermal emission with a Gaussian profile at $v_{\mathrm{lsr}}$ in two sources: DR21(OH) (top) and W43-MM1 (bottom).

detected. Sources with upper limits to the $12 \mu \mathrm{m}$ luminosity or the molecular emission-line flux are not used in the correlation analysis. Applying a two-tailed correlation test for $N=18$ data points (or 7 for water), we reject correlation factors with values smaller than 0.49 (respectively 0.76 ) in absolute value. We also use the method of partial correlations (see Appendix A) to search for biases that could be introduced by the sample selection and extract the most probable real correlations, this allowing us to correct for false correlations induced by real ones (see Rodgers \& Nicewander 1988, for a review of the subject).
Table 10. Correlation matrix for the sample and the $\mathrm{CH}_{3} \mathrm{OH}$ emission analysis.

\begin{tabular}{ccccccccc}
\hline \hline & $M$ & $L$ & $\Delta v$ & $L_{12}$ & $A_{\mathrm{m}}$ & $A_{\mathrm{t}}$ & $X_{\mathrm{m}}$ & $X_{\mathrm{mol}}$ \\
\hline$M$ & 1 & & & & & & & \\
$L$ & 0.50 & 1 & & & & & & \\
$\Delta v$ & 0.45 & 0.49 & 1 & & & & & \\
$L_{12}$ & 0.23 & $\mathbf{0 . 8 0}$ & 0.19 & 1 & & & & \\
$A_{\mathrm{m}}$ & 0.34 & -0.41 & 0.16 & -0.67 & 1 & & & \\
$A_{\mathrm{t}}$ & $\mathbf{0 . 7 0}$ & 0.01 & 0.31 & -0.39 & $\mathbf{0 . 7 6}$ & 1 & & \\
$X_{\mathrm{m}}$ & -0.01 & -0.58 & 0.02 & -0.68 & $\mathbf{0 . 8 8}$ & -0.37 & 1 & \\
$X_{\mathrm{mol}}$ & 0.37 & 0.10 & -0.05 & -0.01 & 0.21 & 0.51 & -0.09 & 1 \\
\hline
\end{tabular}

Notes. Factors under the threshold of confidence are given in italic font, correlations approved by the partial correlation test are in bold font. As the matrix is symmetric, we have only filled its lower part.

\subsection{Search for biases in the sample}

In our systematic search for correlations, we first look for links between "standard variables" (mass, luminosity, and thermal line width and luminosities at $12 \mu \mathrm{m})$. Apart from the thermal line widths, correlations are checked in logarithmic scales. To reliably compare our sources, we need to account for their different distances. To do so, we assume that they all are at a reference distance of $1.7 \mathrm{kpc}$ by applying a correction factor to the flux densities, i.e. measuring monochromatic luminosities at $12 \mu \mathrm{m}$ and comparable methanol maser emissions. No correction is made for beam dilution, as the infrared and maser emissions are known to originate in a region much smaller than the beam size (pointlike emission). We search for correlations with "standard variables" regardless of whether there is a physical reason to expect a correlation: this search acts as a reality check on our method.

Three relations are identified when applying the correlation method. First, a link seems to exist between the mass and the luminosity of the sources $\left(\rho_{M, L}=0.50\right.$, thus $97.5 \%$ of a chance of being real statistically). However, a partial correlation analysis rejects this trend by giving a value under the confidence threshold $\left(\rho_{M L, \Delta v}=0.36\right)$ when keeping $\Delta v$ constant. Second, a weak correlation may exist between thermal line velocity widths and the luminosity of the sources $\left(\rho_{\Delta v, L}=0.49\right)$ but again partial correlation factors reduce this value to 0.34 , by keeping the mass constant. This result has a $16.6 \%$ probability of being obtained by chance, which is too high to claim that a link exists. A third correlation appears between luminosity and mid-IR emission, with $\rho_{L, L_{12}}=0.80$. This trend is clearly confirmed by partial correlation factors, indicating that we can be confident in this result to a level of more than $99.9 \%$ (see Fig. 6).

\subsection{Methanol correlations}

We show in Sect. 5 that two components can be extracted from the methanol line emission: a thermal $\left(A_{\mathrm{t}}\right)$ and a maser one $\left(A_{\mathrm{m}}\right)$. As these two components reflect two different characteristics of the sources, we try to find correlations between them and the intrinsic variables (mass, luminosity, etc.). We also test a possible correlation with the ratio $X_{\mathrm{m}}$ and the abundance $X_{\mathrm{mol}}$. The overall correlation matrix, summarizing the results, is given in Table 10.

The first correlation relates the mid-IR luminosities to the maser component $\left(\rho_{A_{\mathrm{m}}, L_{12}}=-0.67\right)$. The correlation factor is negative, indicating an anti-correlation between these two variables (see Fig. 6). This trend is confirmed by the partial 
Table 11. Correlation matrix for the water emission analysis.

\begin{tabular}{cccccccc}
\hline \hline & $M$ & $L$ & $\Delta v$ & $F$ & $\mathrm{HDO}$ & $\mathrm{H}_{2}^{18} \mathrm{O}$ & $\mathrm{D} / \mathrm{H}$ \\
\hline$M$ & 1 & & & & & & \\
$L$ & 0.28 & 1 & & & & & \\
$\Delta v$ & 0.43 & 0.57 & 1 & & & & \\
$\mathrm{~F}$ & -0.13 & $\mathbf{0 . 7 9}$ & 0.22 & 1 & & & \\
$\mathrm{HDO}$ & 0.06 & 0.38 & -0.22 & 0.71 & 1 & & \\
$\mathrm{H}_{2}^{18} \mathrm{O}$ & 0.00 & 0.46 & -0.18 & 0.80 & $\mathbf{0 . 9 5}$ & 1 & \\
$\mathrm{D} / \mathrm{H}$ & 0.102 & 0.10 & 0.02 & 0.01 & 0.16 & -0.11 & 1 \\
\hline
\end{tabular}

Notes. Factors under the threshold of confidence are given in italic font, correlations approved by the partial correlation test are in bold font. As the matrix is symmetric, we have only filled its low part.

correlation analysis, reducing the correlation factor to -0.65 when the thermal component is assumed to be constant, and giving approximately a $98.5 \%$ of chance of this correlation being real. We note that there is no significant correlation of the integrated (thermal + maser) $\mathrm{CH}_{3} \mathrm{OH}$ line flux in our data. A partial correlation analysis demonstrates that the weakening of the $\mathrm{CH}_{3} \mathrm{OH}$ maser flux with increasing $12 \mu \mathrm{m}$ luminosity is not caused by increasing distance.

In addition, we find two correlations associated with $A_{\mathrm{t}}$. The first one indicates that thermal $\mathrm{CH}_{3} \mathrm{OH}$ emission increases with the mass of the object $\left(\rho_{A_{\mathrm{t}}, M}=0.70\right.$, see Fig. 6). The partial correlation analysis confirms this trend and, at its most extreme, decreases the correlation factor to 0.65 when $L$ is assumed to be constant. There is a probability of lower than $0.4 \%$ that this trend is obtained by chance. The thermal emission is also linked to the maser emission, the correlation factor being equal to 0.76 (see Fig. 6). The study of partial correlation factors slightly decreases the initial value to 0.75 , giving a high chance $(99.9 \%$ statistically) for this result being real.

Three other links, all related to the ratio $X_{\mathrm{m}}$ (see Table 10), are found but rejected by the partial correlation analysis, except the correlation with the maser emission which increases (up to 0.96; this result is obvious as $X_{\mathrm{m}}=A_{\mathrm{m}} / A_{\mathrm{t}}$ ). More precisely, partial correlation factors obtained for other correlations are well below the threshold, with $|\rho|=0.27$ at the maximum, hence there is more than a $27 \%$ chance of obtaining this result by luck. Thus, this ratio is interesting but a bit less relevant than $A_{\mathrm{m}}$.

\subsection{Water correlations}

We now consider the water abundances, including numbers obtained in other MDCs (van der Tak et al. 2006). We also search for possible correlations with the deuteration level D/H. Only the sources where water line emission is detected are included, hence 8 MDCs. As a consequence, the confidence threshold for a real correlation is now 0.756 , assuming a two-tailed test (or two- $\sigma$ test).

First of all, the analysis of biases in this restricted sample reveals, again, a link between the mid-IR luminosity at $12 \mu \mathrm{m}$ $L_{12}$ and the total luminosity $L$ (see Table 11 ). Partial correlation factors confirm this trend with a final correlation factor of 0.79 , hence $98 \%$ of chance of this relation being real. This is a strong indication that these two variables are correlated, even when a smaller sample of sources is chosen.

Another correlation is found, between the mid-IR emission $L_{12}$ and the HDO abundance $\left(\rho_{L_{12}, \mathrm{HDO}}=0.80\right.$, see Table 11$)$. This is not confirmed by the partial correlation analysis, for which we measure a factor beneath the threshold when the $\mathrm{H}_{2}^{18} \mathrm{O}$ abundance is assumed to be constant at $\rho_{L_{12}, \mathrm{HDO}, \mathrm{H}_{2}^{18} \mathrm{O}}=0.58$, hence a $12.9 \%$ chance of having obtained this result with luck, statistically.

The last connection that we find is between the HDO and $\mathrm{H}_{2}^{18} \mathrm{O}\left(\rho_{\mathrm{HDO}, \mathrm{H}_{2}^{18} \mathrm{O}}=0.95\right.$, see Table 11 and Fig. 6$)$. This relation is strong and the partial correlation method confirms this trend by decreasing, at most, the correlation factor to 0.90 when the mid-IR flux density $L_{12}$ is assumed to be constant. This value indicates that our result is real in more than $99.8 \%$ of the cases. This correlation was already noted by eye (Table 8 ) and confirms that the $\mathrm{D} / \mathrm{H}$ ratio is of the same order of magnitude in all studied MDCs.

More globally, our study illustrates that water abundance (hence detection) does not depend on a basic characteristic of HMPOs (such as their mass or luminosity) but needs further investigations in each specific source. This point is discussed in Sect. 8.2.

\section{Discussion}

\subsection{Methanol masers: shocks in embedded objects?}

Our study reveals a clear link between the mid-IR brightness of the 18 MDCs (including the sample by van der Tak et al. 2006) that we have observed and the methanol maser emission at $84.5 \mathrm{GHz}$ (class I maser). Unlike class II masers, which originate in static regions near massive protostars, class I masers are known to occur in extended zones, at some distance from the star formation sites $\left(\sim 10^{4} \mathrm{AU}\right)$, where molecular outflows interact with the surrounding medium (Minier et al. 2005). Cyganowski et al. (2009) study 20 massive young stellar objects observed with interferometry to confirm this behaviour, and claim that methanol class I masers trace the molecular outflows around them.

Molecular outflows are indicators of an accretion process, and are known to decrease in intensity with time as the protostar evolves (low-mass case, see Bontemps et al. 1996). Presenting an early stage of massive star formation, most of MDCs harbour powerful outflows. However, extraction of molecular outflow strength is biased by the unknown orientation and the possible source multiplicity, and no clear drop in strength can be observed between mIRq- and mIRb-MDCs (Beuther et al. 2002a; Marseille et al. 2008). Furthermore, the decrease in power is suspected to occur at a later stage, i.e. in MDCs where an Hir region has developed. As a consequence, the variation in the methanol class I maser emission cannot be linked to a drop in the molecular outflow strength from mIRq to mIRb sources. Like class II masers, class I maser emission needs a very specific environment, with a sufficient spatial extend. Moreover, the detection of multiple velocity components implies that the class I maser sites are localized at the interface between the molecular outflows and the ambient gas. We can assume that mIRq sources contain more favourable zones where this kind of maser emission is more likely to occur.

The origin of the varying mid-IR brightnesses in MDCs remains quite unclear. On the one hand, modelling their spectral energy distribution shows that the mid-IR emission is critically dependent on the line of sight when molecular outflows dig a cavity from which IR emission can escape (Robitaille et al. 2006; van der Tak et al. 2006; Marseille et al. 2008). Thus the variations in mid-IR brightness are orientation-dependent and do not reflect any difference in evolutionary stage. On the other hand, chemical models and observations of sulphur-bearing molecules 
Early evolutionary stages of high-mass star formation: M. G. Marseille et al.
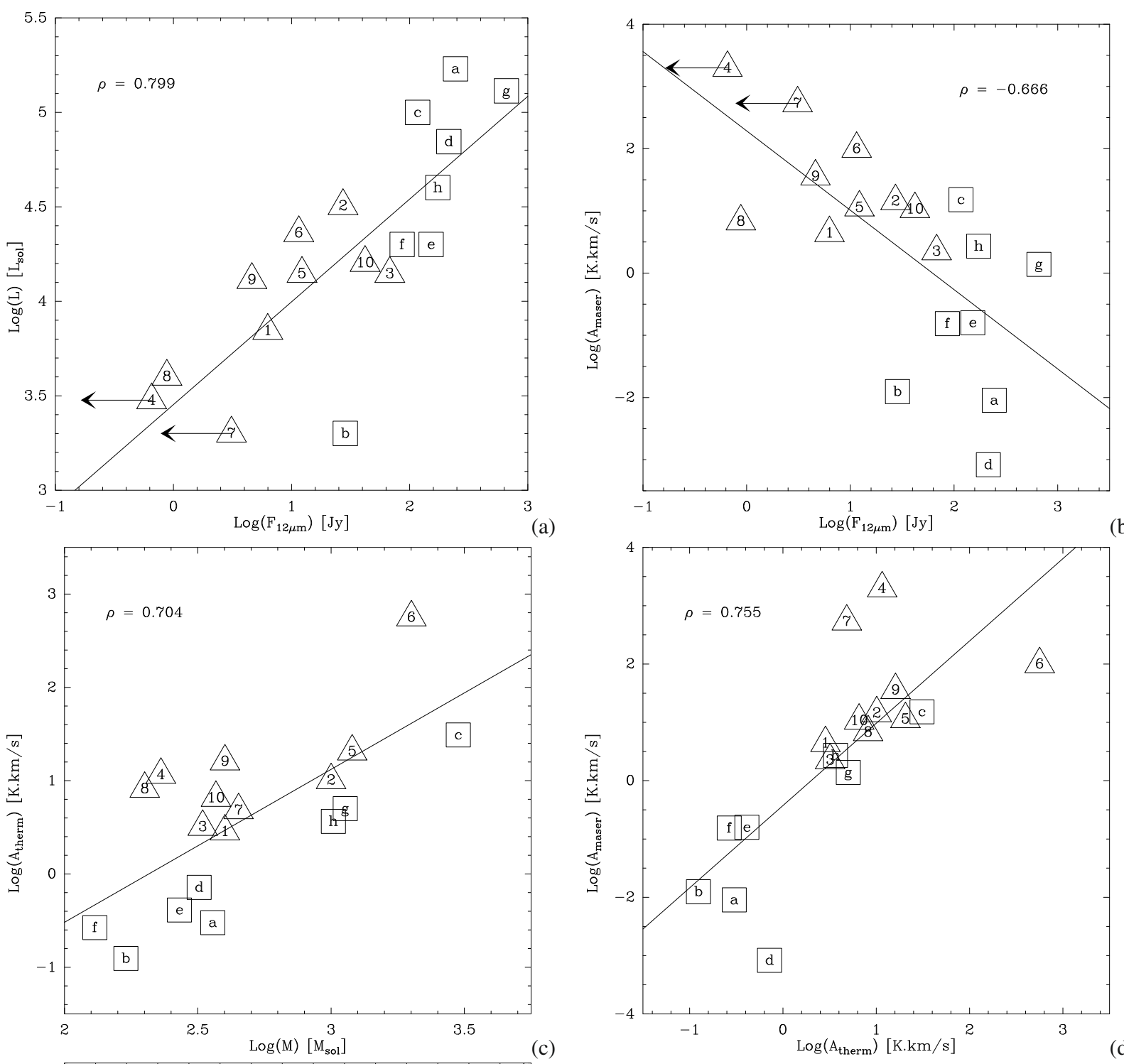

(b)
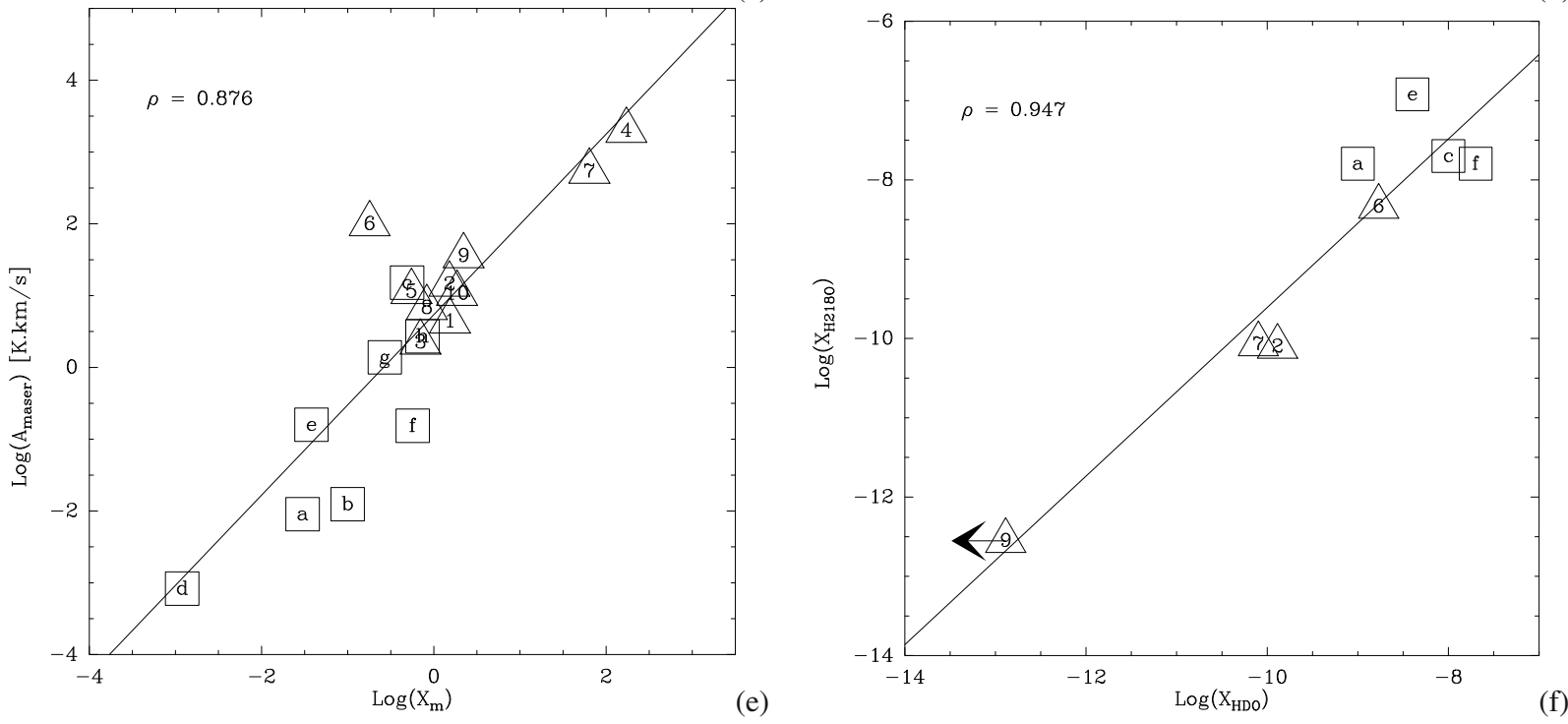

Fig. 6. Correlation plots between a) monochromatic luminosities at $12 \mu \mathrm{m}$ and source luminosity; b) monochromatic luminosities at $12 \mu \mathrm{m}$ and maser component of $\mathrm{CH}_{3} \mathrm{OH}$ emission line; $\mathbf{c}$ ) masses of HMPOs and the thermal component of $\mathrm{CH}_{3} \mathrm{OH}$ emission lines; $\mathbf{d}$ ) the thermal and the maser component of $\mathrm{CH}_{3} \mathrm{OH}$ emission lines; e) the maser-to-thermal emission ratio and the maser emission; $\mathbf{f}$ ) the molecular abundances of $\mathrm{HDO}$ and $\mathrm{H}_{2}^{18} \mathrm{O}$. Sources of our sample are labelled with numbers (1 to 10), sources studied by van der Tak et al. (2006) are labelled with letters (a to h). Solid line: best linear fit obtained with a least squares method. 
or cold gas tracers such as $\mathrm{N}_{2} \mathrm{H}^{+}$seem to indicate that mid-IR brighter sources are more evolved and warmer, relative to more quiet ones (Reid \& Matthews 2008; Marseille et al. 2008; Herpin et al. 2009).

The anti-correlation that we find between the $12 \mu \mathrm{m} \mathrm{lu}$ minosity and the maser emission makes the situation clearer. Indeed, the hypothesis that the variations in mid-IR brightness are due to orientation differences cannot explain this trend, as the maser emission is not affected by the line of sight direction. Furthermore, the multiple velocity components would be more easily detected if the molecular outflows, i.e. the cavity from where the mid-IR is emitted, were to follow the line of sight (case of mIRb-MDCs). However, we observe the opposite trend. A valid interpretation defines mIRq-HMPOs as embedded objects, in which the envelope mainly interacts with outflows. Here, the term "embedded" must be defined to avoid confusion. Our study has found that the envelope masses of MDCs are not linked to their mid-IR brightness, hence are not sufficient to determine wether they are embedded or not. Taking into account the material distribution, the SED modelling of MDCs shows that their spherically symmetric distribution is responsible for their mid-IR emission from MDCs (Robitaille et al. 2006; Marseille et al. 2008). In this sense, our study shows that mIRqMDCs are embedded sources. Bright mid-IR objects may thus have more flattened envelopes than weak mid-IR ones. From an observational point of view, this idea is supported by the Kurtz et al. (2004) study of the IRAS $23385+6053$ source where maser emission is detected around the source velocity (at $-49.7 \mathrm{~km} \mathrm{~s}^{-1}$ and $-51.7 \mathrm{~km} \mathrm{~s}^{-1}$ ). The conclusion of this study is that this observation can be explained by accretion shocks near the central object. This could explain also what we observe for W43-MM1, where strong clues of infall toward this source have been detected (Herpin et al. 2009).

Nevertheless, an embedded phase does not imply that they correspond to an earlier stage of evolution. For example, this characteristic can be a direct consequence of the initial conditions of the MDC formation. However, our results support a scenario in which the initial collapsing clump, radially concentrated, is disrupted by the molecular bipolar outflows during the accretion phase. Moreover, the mid-IR brightness and evolutionary stage of MDCs are linked.

\subsection{The origin of water emission}

Previous results on $\mathrm{HDO}$ and $\mathrm{H}_{2}^{18} \mathrm{O}$ detections in mIRb-MDCs obtained by van der Tak et al. (2006) demonstrated that these objects are not systematically strong $\mathrm{H}_{2} \mathrm{O}$ emitters. Our study has a similar detection rate (just below $\sim 50 \%$ ) and does not show any evidence of an emission enhancement from $\mathrm{mIRq}$ to $\mathrm{mIRb}$ sources. This contradicts the hypothesis that warmer objects should be stronger emitters. Indeed, a warmer environment should increase the release of water in the gas phase from the ice on dust grains. Thus, the origin of the strong line emission of water species in particular sources cannot be linked to the "quiet" versus "bright" scheme. Each source must be treated individually.

The IRAS 18089-1732 MDC is the most luminous object in the mIRq sample $\left(3.2 \times 10^{4} L_{\odot}\right)$, and is also the source where the turbulence level is the highest $\left(v_{\mathrm{T}}=1.2-2.6 \mathrm{~km} \mathrm{~s}^{-1}\right)$. The discernible methanol class I maser emission in this object $\left(X_{\mathrm{m}}=\right.$ 1.42 ) is indicative of interactions between the molecular outflows and the envelope. We have shown that the abundance of $\mathrm{H}_{2} \mathrm{O}$ and $\mathrm{HDO}$ is high enough to be detected (see Table 8). We thus conlude that, in IRAS 18264-1152, the water abundance enhancement is caused by the large amount of energy available from luminosity, gas turbulence, and molecular outflows (shocks). In addition, the detection of unexpected dimethyl-ether emission in the $\mathrm{H}_{2}^{18} \mathrm{O}$ spectrum suggests that complex chemical components are released in the gas phase, including the water ice. We propose that the enhancement originates in a hot molecular core whose presence may also explain why water is detected in this source.

In W43-MM1, many molecular rotational transitions have been detected, covering a wide range of energy (Herpin et al. 2009). The large amount of detected species, even unexpected complex molecules, are indicative of an extended hot molecular core, and make this source of particular interest. Moreover, this region is suspected to be in global infall (Motte et al. 2003; Herpin et al. 2009). This very likely explains the very high abundances (see Table 9) in this source: the ice on dust grains must have evaporated, increasing the water abundance to $2 \times 10^{-6}$.

Water line profiles towards DR21(OH) exhibit a double velocity component that may be linked to powerful bipolar outflows, the water emission being produced by shocks between molecular outflows and the massive envelope. This is confirmed by the strong methanol class I maser emission $\left(X_{\mathrm{m}}=1.58\right)$, and wherein three components are identified, but the velocity components observed in $\mathrm{H}_{2} \mathrm{O}$ and $\mathrm{HDO}$ profiles differ from the velocity components of the maser emission (see Table 5). In addition, this scenario (increase in the amount of water species in the gas phase due to shocks) should apply to IRAS 18151-1208-MM2, the most powerful methanol maser emitter $\left(X_{\mathrm{m}}=13.9\right)$, but no HDO and $\mathrm{H}_{2}^{18} \mathrm{O}$ emission is detected. Hence, we conclude that shocks in DR2 $1(\mathrm{OH})$ are not the origin of the abundance enhancement of water. DR21(OH) is one of three sources where unexpected detections of dimethyl-ether have been observed, showing that a hot molecular core takes place there. This is the best argument to explain the water abundance in this source.

On the other hand, HDO is detected in the source NGC 7538S, where there is no indication of a hot core. Furthermore, this object is not a strong methanol class I maser emitter $\left(X_{\mathrm{m}}=0.21\right)$, showing that no strong shocks occur inside it. The origin of the water detection is then more difficult to explain. In addition, our modelling of NGC 7538 S shows that the water abundance is not particularly high compared to upper limits derived for other sources (see Table 8), even if this MDC has the highest temperature of the sample, well above the others $(\langle T\rangle=38.7 \mathrm{~K})$. This high temperature is caused by the small size of this source $(0.05 \mathrm{pc})$ combined with its high luminosity $\left(1.3 \times 10^{4} L_{\odot}\right)$. Therefore, we suppose that the emission of water species in NGC 7538S, while not as abundant as those in hot cores, is stronger due to this high temperature. However, the derived abundances are consistent with an abundance jump of $1 \times 10^{4}$ in the hottest part of NGC 7538S. Indeed, we obtained low but almost equivalent values when considering either low or high energy level transitions $\left(\sim 1 \times 10^{-13}\right)$. Another possibility would be that NGC 7538 S harbors a brand new hot core. In that case, primal ices forming on dust grains in the atomic phase of diffuse clouds are strongly deuterated by the formation mechanism (Lipshtat et al. 2004; Cazaux et al. 2008). This could explain why HDO is detected while $\mathrm{H}_{2}^{18} \mathrm{O}$ is not, these primal ices being first desorbed when a hot core starts to develop.

Our study of a large sample of MDCs permits us to conclude that the water detection in these objects is not linked to their mid-IR brightness, i.e. a different evolutionary stage or a different distribution of materials. In addition, it shows that water detection is associated with a hot molecular core. This seems 
Early evolutionary stages of high-mass star formation: M. G. Marseille et al.

reasonable as in the gas phase hot cores are known to release complex molecules that are detected, while ice on dust grains evaporates. In rare cases, such as NGC 7538S, water line emission may be detected due to a globally high temperature in the source.

\section{3. $m I R q$ vs $m I R b$ : review of common and different properties}

Several clues show that mIRq- and mIRb-MDCs have similar properties. First, previous work on large samples of MDCs indicate that the velocity widths of emission lines are dominated by gas turbulence $\left(v_{\mathrm{T}}=0.8-2.9 \mathrm{~km} \mathrm{~s}^{-1}\right)$, whose corresponding width is much higher than the thermal width (Beuther et al. 2002b; Motte et al. 2007). Our observations confirm this result, and that this high turbulence may be the consequence of the initial cloud collapse, as suspected in large-scale filaments (e.g. DR21 in Cygnus X, see Motte et al. 2007; or the Ophiuchus main cloud, see André et al. 2007). In this context, the turbulence is supposed to dissipate by means of ambipolar diffusion while the MDC evolves, leading to a quasi-static collapse (see Ward-Thompson et al. 2007, for a review). Our study shows that the turbulence does not vary between mIRq- and mIRb-MDCs, indicating that their evolutionary stages are similar. We also note that the range of masses in our sample (200-2000 $M_{\odot}$ ) has no impact on the mid-IR brightness of the sources. To first order, the most massive sources should have a higher opacity at midIR wavelengths. Our study contradicts this simple view. The emission process of mid-IR is complex in MDCs, and must include other parameters. Previous work on the SED modelling of MDCs show that the density distribution plays a major role in this issue (Robitaille et al. 2006; Marseille et al. 2008).

Our work has revealed that the water abundance in MDCs is independent of their mid-IR brightness. The detection ratio in $\mathrm{mIRq}$ and $\mathrm{mIRb}$ sources is the same (slightly below 50\%). Water abundances derived are similar, with a wide range of values in the external cold parts of the objects (between $\sim 1 \times 10^{-13}$ and $\sim 1 \times 10^{-9}$ ). From our study, we conclude that most objects for which water species are detected contain a more or less extended hot molecular core. Finally, we have found that $\mathrm{mIRq}$ and $\mathrm{mIRb}$ sources share the same deuteration ratio $\mathrm{D} / \mathrm{H} \sim(5-40) \times 10^{-4}$. As already reported in van der Tak et al. (2006), this value is one hundred times higher than the interstellar ratio, and confirms that the molecular emission observed is coming from evaporated ices.

These results illustrate the difficulty in differentiating between the $\mathrm{mIRq}$ and $\mathrm{mIRb}$ classes. The origin of this distinction is linked to the class 0 and class I definitions for low-mass protostars (André et al. 1993), where the mid-IR luminosity is compared to the overall luminosity of the sources. A ratio $L_{\lambda<25 \mu \mathrm{m}} / L_{\text {bol }}<0.3 \%$ indicates a class 0 object, and a class I object in the opposite case. This definition was extended to MDCs where a similar behaviour at mid-IR wavelengths was observed, and "quiet" sources were suspected to represent an earlier stage of the massive star formation evolution. However, it appears more and more clearly that directly applying definitions from low-mass protostars to high-mass star-forming regions is unwise and may not be adapted to correctly transcribe different evolutionary stages. This is caused by the multiplicity of MDCs, that are now identified as massive proto-clusters, but also to the presence of powerful outflows, hot molecular cores and suspected infall (Motte et al. 2003; van der Tak et al. 2006; Leurini et al. 2007b; Ward-Thompson et al. 2007; Marseille et al. 2008; Herpin et al. 2009). Furthermore, we show that the observed variation in mid-IR brightness seems to be associated with the matter distribution around the protocluster, being more or less spherically symmetric.

Interpreting mIRq sources as more embedded objects supports the idea that they represent an earlier stage of the massive star formation. Indeed, with the hypothesis that MDCs start in a deeply embedded phase where matter is then flattened by powerful outflows, as the methanol class I maser trend seems to illustrate, the evolutionary sequence from mIRq- to mIRb-MDCs is still valid.

\section{Conclusions}

We summarize the main conclusions of our study:

1. Using a correlation method, we have derived a link between the methanol class I maser emission and the mid-IR luminosity of the MDCs that we observed. We propose that this link can be explained by the mIRq sources being more deeply embedded, i.e. having a more spherically symmetric distribution of their envelope material, creating a favourable environment for the creation of these masers.

2. The detection of line emission from $\mathrm{H}_{2} \mathrm{O}$ and $\mathrm{HDO}$ is linked to a hot molecular core, independent of the mid-IR brightness.

3. The study of the overall sample has demonstrated that MDCs have many common properties: their size $(0.1 \mathrm{pc})$, mass ( 200-2000 $\left.M_{\odot}\right)$, luminosity $\left(\sim 1 \times 10^{4} L_{\odot}\right)$, turbulent velocity of the gas $\left(v_{\mathrm{T}}=1-3 \mathrm{~km} \mathrm{~s}^{-1}\right)$, incidence of hot cores $(\$ 50 \%)$, and deuteration level $\left(\mathrm{D} / \mathrm{H} \sim 5-40 \times 10^{-4}\right)$. This implies the idea that these objects can be treated as a single class, and that they constitute a cluster of protostars, some of them being massive.

4. The classification of MDCs is complex due to their multiplicity and the multiple physical processes (independent of their evolutionary stage) that occur inside, which have a strong impact on observations, i.e. hot molecular core presence, and powerful outflows, suspected collapse. We finally suggest that the mid-IR "quiet" and "bright" classification needs more investigation but is still relevant when we assume that $\mathrm{mIRq}$ sources represent an earlier stage of massive star formation than $\mathrm{mIRb}$ ones.

In the near future, further investigation of the mIRq versus mIRb-MDCs evolutionary stage problem will be possible, particularly by means of direct water line detections with the Herschel-HIFI instrument and the high-angular resolution of present (IRAM, SMA, CARMA) and future (ALMA) interferometers. These may provide us with the final arguments permitting us to find a coherent classification, by age, of the MDCs, hence allowing us to describe a complete evolution scenario leading to the formation of massive stars.

Acknowledgements. We thank the editor, Malcolm Walmsley, for many comments that improved the quality of the manuscript.

\section{Appendix A: Elements of correlation theory}

For more detail and a global review of correlation factor usage, we refer to Rodgers \& Nicewander (1988). 


\section{A.1. Correlation factor definition}

Given two variables $x$ and $y$ with $N$ elements $x_{i}$ and $y_{i}$, the correlation factor $\rho_{x, y}$ between these two variables is measured to be

$\rho_{x, y}=\frac{\sum_{i=1}^{N}\left(x_{i}-\mu_{x}\right)\left(y_{i}-\mu_{y}\right)}{N^{2} \sigma_{x} \sigma_{y}}$,

where

$\mu_{x}=\frac{1}{N} \sum_{i=1}^{N} x_{i}$

$\sigma_{x}=\frac{1}{N} \sum_{i=1}^{N}\left(x_{i}-\mu_{x}\right)^{2}$.

A correlation factor has a value between -1 and 1 , a positive value indicating a correlation between the two variables and a negative value indicating an anti-correlation. If two variables are distributed randomly following a normal law, the density of probability $P(\rho)$ of finding a correlation factor between $\rho$ and $\rho+\mathrm{d} \rho$ is given by

$P(\rho)=\frac{1}{\sqrt{\pi}} \frac{\Gamma\left(\frac{v+1}{2}\right)}{\Gamma\left(\frac{v}{2}\right)}\left(1-\rho^{2}\right)^{(v-2) / 2}$,

where $\Gamma$ is the classic gamma function, and $v=N-2$ where $N$ is the number of elements (see Fig. A.1 for plots at different $N$ ). Thus, the absolute value of the correlation gives, as a function of the number of elements $N$, a percentage of the distribution $\left(x_{i}, y_{i}\right)$ obtained by chance. The higher the absolute value of the correlation factor is, the lower this percentage. As an example, a correlation factor of -0.5 obtained with 20 elements has a good chance $(97.5 \%)$ of being real. With only 6 elements, the same correlation factor has a lesser chance $(68.8 \%)$ of being real.

One can note that the $P(\rho)$ function peaks more and more around $\rho=0$ while $N$ increases. More precisely, the standard deviation of the $P(\rho)$ distribution is given by

$\sigma^{2}=\left\langle P^{2}\right\rangle-\langle P\rangle^{2}=\frac{1}{N-1}$.

It is then common practise to perform a two-tailed (two-sigma) test to reject the null-hypothesis, i.e. that the $\left(x_{i}, y_{i}\right)$ points does not follow a distribution of two random variables. This permits us to define a correlation factor threshold $\rho_{\mathrm{t}}$ equal to $2 \sigma$ to claim wether a correlation has a good chance of being real or not

$|\rho|>\rho_{\mathrm{t}}=\frac{2}{\sqrt{N-1}}$.

This formula justifies that $N \geq 6$, which is a necessary criterion to obtain a threshold lower than 1 . In the case of our study, we used the two-sigma test to discriminate between true and false correlation.

\section{A.2. Partial correlations}

When studying multiple variables at the same time, it is very often useful to have a clear view of the links between the variables, and first determine wether they exist or not. Existence of these links can be identified by determining the correlation factors of pairs of variables (see section above).
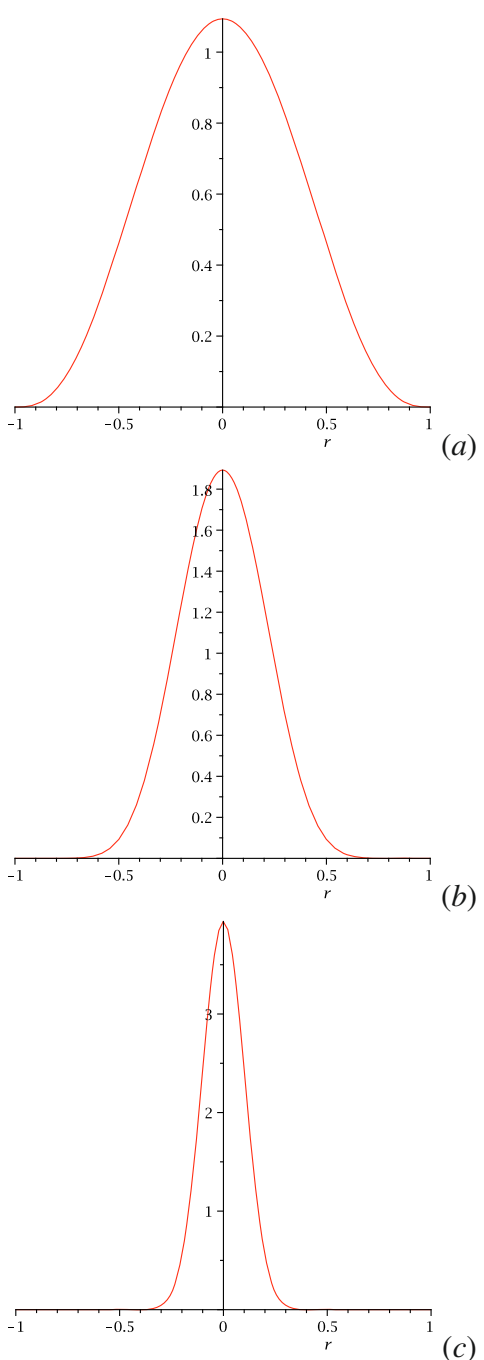

(c)

Fig. A.1. Plots of the $P(\rho)$ with different number of elements on the sample studied: a) with $N=10$, b) with $N=25$, and c) with $N=100$.

Unfortunately, the use of correlation factors restricts the study to pairs of variables, which can lead to the identification of false links, i.e. two variables that seems to be correlated when a third variable is the true origin of this link. One can take the example of a sample of pupils of all ages. A raw study of correlations between their ages, weights, and scientific levels will certainly make establish a false link: between the weight and the scientific level. Indeed, we can easily understand that their ages are at the origin of their growth, physically and intellectually. For a sample in which this logical deductions would be impossible, how can we distinguish true from false correlations?

The use of partial correlations permits us to avoid this issue. Giving a set of three variables $x, y$, and $z$, partial correlation $\rho_{x y, z}$ derives the correlation factor between two variables (for example $x$ and $y$ ), assuming that the third one $z$ remains constant during the measurements. The partial correlation factor is then given by

$\rho_{x y, z}=\frac{\rho_{x y}-\rho_{x z} \rho_{y z}}{\sqrt{\left(1-\rho_{x z}^{2}\right)\left(1-\rho_{y z}^{2}\right)}}$.

If the partial correlation factor obtained is below the confidence threshold chosen or has a different sign from the standard correlation factor, then the link between the two variables is false. 
Early evolutionary stages of high-mass star formation: M. G. Marseille et al.

For a large set of variables $\left\{X_{i}\right\}$, all the links obtained by standard correlations factors $\rho_{X_{i}, X_{j}}$ must be verified with a systematic checking of all partial correlations associated with it, i.e. all $\rho_{X_{i} X_{j}, X_{k \neq i, j\}}}$ must be above the threshold of confidence and have the same sign as $\rho_{X_{i}, X_{j}}$.

\section{References}

André, P., Ward-Thompson, D., \& Barsony, M. 1993, ApJ, 406, 122 André, P., Belloche, A., Motte, F., \& Peretto, N. 2007, A\&A, 472, 519 Barvainis, R., \& Deguchi, S. 1989, AJ, 97, 1089

Belloche, A., Garrod, R. T., Müller, H. S. P., et al. 2009, A\&A, 499, 215

Beuther, H., Schilke, P., Gueth, F., et al. 2002a, A\&A, 387, 931

Beuther, H., Schilke, P., Menten, K. M., et al. 2002b, ApJ, 566, 945

Beuther, H., Schilke, P., Sridharan, T. K., et al. 2002c, A\&A, 383, 892

Beuther, H., Walsh, A., Schilke, P., et al. 2002d, A\&A, 390, 289

Błaszkiewicz, L., \& Kus, A. J. 2004, A\&A, 413, 233

Bonnell, I. A., Bate, M. R., Clarke, C. J., \& Pringle, J. E. 1997, MNRAS, 285, 201

Bonnell, I. A., Bate, M. R., Clarke, C. J., \& Pringle, J. E. 2001, MNRAS, 323, 785

Bonnell, I. A., Bate, M. R., \& Zinnecker, H. 1998, MNRAS, 298, 93

Bontemps, S., Andre, P., Terebey, S., \& Cabrit, S. 1996, A\&A, 311, 858

Bontemps, S., Motte, F., Csengeri, T., \& Schneider, N. 2010, A\&A [0909.2315]

Boonman, A. M. S., Doty, S. D., van Dishoeck, E. F., et al. 2003, A\&A, 406, 937

Caswell, J. L., Vaile, R. A., Ellingsen, S. P., \& Norris, R. P. 1995, MNRAS, 274, 1126

Caswell, J. L., Yi, J., Booth, R. S., \& Cragg, D. M. 2000, MNRAS, 313, 599

Cazaux, S., Caselli, P., Cobut, V., \& Le Bourlot, J. 2008, A\&A, 483, 495

Cernicharo, J., Thum, C., Hein, H., et al. 1990, A\&A, 231, L15

Cyganowski, C. J., Brogan, C. L., Hunter, T. R., \& Churchwell, E. 2009, ApJ, 702,1615

Draine, B. T., \& Lee, H. M. 1984, ApJ, 285, 89

Edris, K. A., Fuller, G. A., \& Cohen, R. J. 2007, A\&A, 465, 865

Elitzur, M. 1992, Astronomical masers Astrophysics and Space Science Library, 170

Girart, J. M., Beltrán, M. T., Zhang, Q., Rao, R., \& Estalella, R. 2009, Science, 324,1408

Guilloteau, S., \& Lucas, R. 2000, in Imaging at Radio through Submillimeter Wavelengths, ed. J. G. Mangum, \& S. J. E. Radford, ASP Conf. Ser., 217, 299

Harwit, M., Neufeld, D. A., Melnick, G. J., \& Kaufman, M. J. 1998, ApJ, 497, L105+

Haschick, A. D., Baan, W. A., \& Menten, K. M. 1989, ApJ, 346, 330
Henriksen, R., Andre, P., \& Bontemps, S. 1997, A\&A, 323, 549 Herpin, F., Marseille, M., Wakelam, V., Bontemps, S., \& Lis, D. C. 2009, A\&A, 504,853

Hogerheijde, M. R., \& van der Tak, F. F. S. 2000, A\&A, 362, 697 Kurtz, S., Hofner, P., \& Álvarez, C. V. 2004, ApJS, 155, 149 Leurini, S., Beuther, H., Schilke, P., et al. 2007a, A\&A, 475, 925 Leurini, S., Beuther, H., Schilke, P., et al. 2007b, A\&A, 475, 925 Lipshtat, A., Biham, O., \& Herbst, E. 2004, MNRAS, 348, 1055

Marseille, M., Bontemps, S., Herpin, F., van der Tak, F. F. S., \& Purcell, C. R. 2008, A\&A, 488, 579

McKee, C. F., \& Tan, J. C. 2003, ApJ, 585, 850

Minier, V., Burton, M. G., Hill, T., et al. 2005, A\&A, 429, 945

Molinari, S., Brand, J., Cesaroni, R., \& Palla, F. 1996, A\&A, 308, 573

Molinari, S., Testi, L., Brand, J., Cesaroni, R., \& Palla, F. 1998, ApJ, 505, L39

Molinari, S., Faustini, F., Testi, L., et al. 2008, A\&A, 487, 1119

Motte, F., Schilke, P., \& Lis, D. C. 2003, ApJ, 582, 277

Motte, F., Bontemps, S., Schilke, P., et al. 2007, A\&A, 476, 1243

Reid, M. A., \& Matthews, B. C. 2008, ApJ, 675, 1343

Robitaille, T. P., Whitney, B. A., Indebetouw, R., Wood, K., \& Denzmore, P. 2006, ApJS, 167, 256

Rodgers, J. L., \& Nicewander, W. A. 1988, The American Statistician, 42, 59

Sandell, G., Wright, M., \& Forster, J. R. 2003, ApJ, 590, L45

Shu, F. H., Adams, F. C., \& Lizano, S. 1987, ARA\&A, 25, 23

Shu, F., Najita, J., Galli, D., Ostriker, E., \& Lizano, S. 1993, in Protostars and Planets III, ed. E. H. Levy, \& J. I. Lunine, 3

Sridharan, T. K., Beuther, H., Schilke, P., Menten, K. M., \& Wyrowski, F. 2002, ApJ, 566, 931

Szymczak, M., Kus, A. J., \& Hrynek, G. 2000, MNRAS, 312, 211

Val'tts, I. E., Ellingsen, S. P., Slysh, V. I., et al. 2000, MNRAS, 317, 315

van der Tak, F. F. S., van Dishoeck, E. F., Evans, I. N. J., Bakker, E. J., \& Blake, G. A. 1999, ApJ, 522, 991

van der Tak, F. F. S., van Dishoeck, E. F., Evans, I. N. J., \& Blake, G. A. 2000a, ApJ, 537, 283

Van der Tak, F. F. S., van Dishoeck, E. F., \& Caselli, P. 2000b, A\&A, 361, 327

van der Tak, F. F. S., Boonman, A. M. S., Braakman, R., \& van Dishoeck, E. F. 2003, A\&A, 412, 133

van der Tak, F. F. S., Walmsley, C. M., Herpin, F., \& Ceccarelli, C. 2006, A\&A, 447, 1011

van Dishoeck, E. F., \& Helmich, F. P. 1996, A\&A, 315, L177

Wakelam, V., Castets, A., Ceccarelli, C., et al. 2004, A\&A, 413, 609

Ward-Thompson, D., André, P., Crutcher, R., et al. 2007, in Protostars and Planets V, ed. B. Reipurth, D. Jewitt, \& K. Keil, 33

Wolf, S., Henning, T., \& Stecklum, B. 1999, A\&A, 349, 839

Yorke, H. W., \& Sonnhalter, C. 2002, ApJ, 569, 846

Zapata, L. A., Rodríguez, L. F., Ho, P. T. P., Beuther, H., \& Zhang, Q. 2006, AJ, 131,939

Zinnecker, H., \& Yorke, H. W. 2007, ARA\&A, 45, 481 